\title{
User-Centered Design of an Attitude-Aware Controller for Ground Reconnaissance Robots
}

\author{
Amber M. Walker \\ United States Military Academy \\ David P. Miller \\ University of Oklahoma \\ and \\ Chen Ling \\ University of Akron
}

\begin{abstract}
Warfighter safety can be significantly increased by offloading critical reconnaissance and surveillance missions to robotic assets. The subtleties of these tasks require significant operator involvement-usually carried out locally to the robot's deployment. Human soldiers use gestures to communicate movements and commands when engaged in this type of task. While considerable work has been done with robots visually observing humans to interpret their gestures, we propose a simpler, more field-appropriate system that allows robot operators to use their natural movements and gestures (via inertial measurement units [IMUs]) to teleoperate a robot while reducing the physical, as well as the cognitive, load on the soldier.

This paper describes an operator control interface implemented on a smartphone, in contrast to the proprietary robot controllers typically used. The controller utilizes the device's IMUs, or attitude sensors, to bypass the touchscreen while accepting user input via gestures; this addresses a primary concern for gloved users in dirty environments where touchscreens lack reliability. We also propose that it provides a less visually-intense alternative for control, freeing up the soldier's cognitive load toward other functions.

We present details of the attitude-based control software, as well as the design heuristics resulting from its iterative build-test-rebuild development. Additionally, results from a set of user studies are presented, showing that as a controller, this technique performs as well, or better than, other screenbased control systems, even when ignoring its advantages to gloved users. Twenty-five users were recruited to assess usability of these attitude-aware controls, testing their suitability for both driving and camera manipulation tasks. Participants drove a small tracked robot on an indoor course using the attitude-aware controller and a virtual [touchscreen] joystick, while metrics regarding performance, mental workload, and user satisfaction were collected. Results indicate that the tilt controller is preferred by $64 \%$ of users and performs equally as well, if not better to the alternative, on most performance metrics. These results support the development of a smartphone-based control option for military robotics, with a focus on more physical, attitude-based input methods that overcome deficiencies of current touch-based systems, namely lack of physical feedback, high attention demands, and unreliability in field environments.
\end{abstract}

Keywords: Human-robot interaction, user-centered design, field robotics, handheld control, interface modality

Authors retain copyright and grant the Journal of Human-Robot Interaction right of first publication with the work simultaneously licensed under a Creative Commons Attribution License that allows others to share the work with an acknowledgement of the work's authorship and initial publication in this journal.

Journal of Human-Robot Interaction, Vol. 4, No. 1, 2015, Pages 30-59. DOI 10.5898/JHRI.4.1.Walker 


\section{Introduction}

As specialized field robots, military unmanned systems have been designed to reliably complete a variety of tasks in unstructured and unpredictable operating environments while remaining immune to fatigue, disease, emotion, and environmental discomfort (Blitch, 2003; Tilbury \& Ulsoy, 2011). To date, the most commonly deployed unmanned ground vehicles (UGVs) are found in explosive ordnance disposal, primarily due to their success in saving lives by keeping soldiers out of harm's way. As a result of this "survivability," UGVs are now being used (or developed) in support of several other missions: reconnaissance and surveillance, logistics and support, communications, and direct combat (Antal, 2009).

To support this evolving mission set, robots are being miniaturized, resulting in more portable, man-packable systems. This serves to increase deployability, enhancing their worth as small-unit reconnaissance and search and rescue vehicles. In these missions, UGVs are employed almost entirely out of line of sight, requiring teleoperation, where users rely on video feedback from one or more onboard cameras to navigate and conduct surveillance. Since even sophisticated UGVs cannot adequately discriminate between friend and foe, or make decisions regarding complex situations-on the battlefield and beyond (Antal, 2009)-the user interface is a critical tool enabling the operator to make these decisions via tele-presence. Interestingly, while robots have gotten increasingly smaller, efforts to shrink the operator interface have not proceeded apace.

Weight is, by far, one of the military's biggest enemies. Soldiers carry their mission load on their back-a load that quickly becomes excessive as equipment improvements/solutions meant to aid in soldier tasks actually hinder movement, putting lives at risk. Loads include not only basic equipment, such as assault pack, weapons and ammunition, food and water, but also clothing and body armor, which alone exceeds 25 pounds (Center for Army Lessons Learned, Task Force Devil, 2003). Adding communications equipment, robot platforms, and the batteries to power them easily eats up the remaining space (and $72 \mathrm{lbs}$. of carrying capacity (Department of the Army, 1990)) of dismounted troops. In fact, the Center for Army Lessons Learned estimates the real-time combat load of light Infantry Riflemen at closer to $95 \mathrm{lbs}$., which is currently in violation of Army doctrine (Center for Army Lessons Learned, Task Force Devil, 2003). The study identifies two major ways to meaningfully reduce this combat load: 1) prioritize the design of lighter soldier systems, and 2) off-load any and all equipment not immediately necessary to firefights onto alternate forms of transportation. Given this, the current operator control units presented in Section 1.1 require further and immediate improvement-namely lighter weight, highly capable device hardware, such as smartphones. To put it in context, the Apple iPhone 6 weighs only $4.55 \mathrm{oz}$. (Apple, 2014).

\subsection{Current Operator Control Units (OCUs)}

For many years, robot control has been achieved via laptop-based systems originally designed for desktop use then rudimentarily converted for field operations (see Fig. 1a). Robots have since gotten smaller, users have become more mobile, and yet the laptop OCU remains as a cumbersome control option. Various versions exist, whether they be worn around the user's neck or carried in a backpack with a tethered handheld component. Aside from their size, many of these systems are inherently complicated for dismounted users, whose demands require more simplicity.

The industry boasts few examples of portable, handheld operator control unit technologies, and even fewer that eschew the standard single-mode tactile joystick. While recently updated controllers have addressed portability, manufacturers appear to be making concerted efforts to avoid touchscreen interfaces, given the uncertainty surrounding their successful adoption for field use. Recon Robotics currently deploys their Throwbot ${ }^{\circledR}$ XT with a simple handheld controller where video and joystick are housed on a device weighing 1.6 pounds (Recon Robotics, 2012) (Fig. 1b); iRobot has upgraded to a small, two-pound handheld controller with 5" LED screen for their FirstLook ${ }^{\circledR}$ robot (iRobot 
Corporation, 2012) (Fig. 1c); and Applied Research Associates (ARA) uses something similar for their Pointman ${ }^{\circledR}$ robot (Applied Research Associates, 2011) (Fig. 1d).

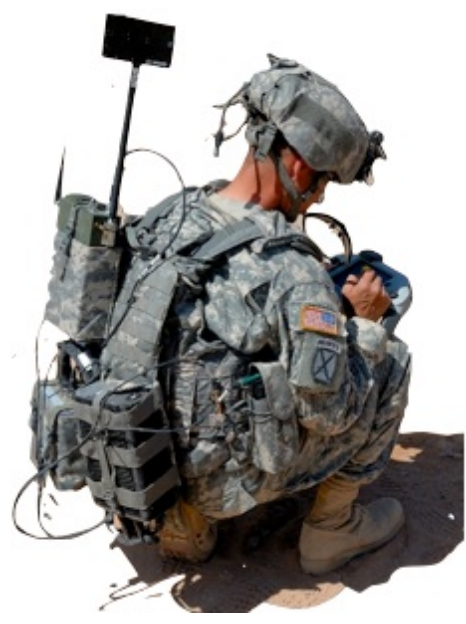

(a) Laptop-based Controller

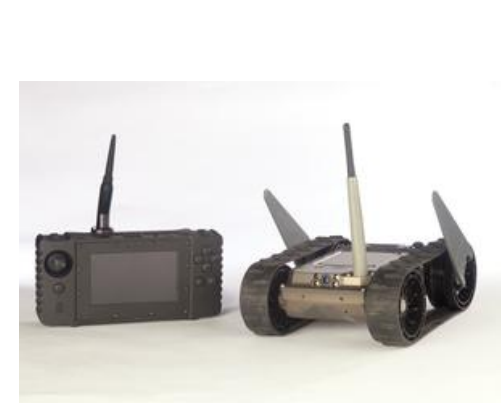

(c) iRobot FirstLook Controller

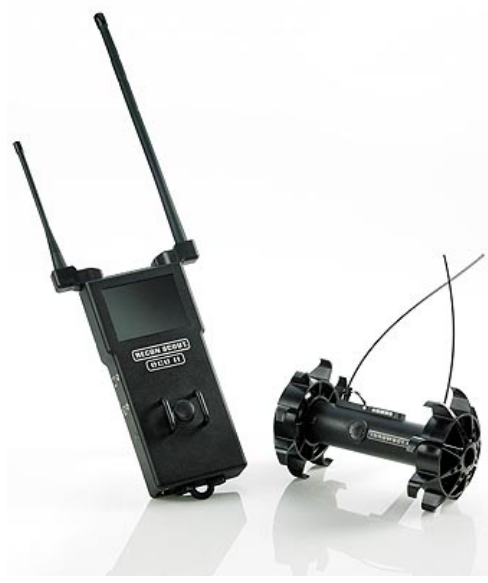

(b) Recon Robotic's Throwbot Controller

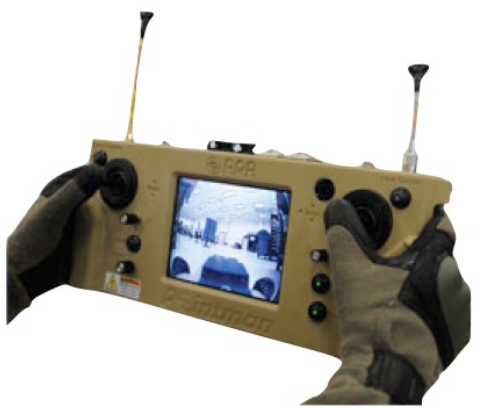

(d) ARA Pointman Controller

Figure 1. Operator Control Unit (OCU) Examples

\subsection{Background \& Motivation}

Part of the delay in adopting newer, sleeker OCU technologies results from the bureaucratic nature of military procurement combined with independent (i.e. not clearly outlined by the "consumer") research and development undertaken by government contractors. During Desert Storm, the Army used legacy techniques for moving (large) units on the battlefield and identified mobile networks, specifically, as a limiting factor to the speed (and tenacity) of engagement (Erwin, 2001). This prompted the Army, in the decades following that conflict, to accelerate efforts toward technological superiority, using more commercially available "off the shelf" items to keep pace with information technology developments in the civilian sector. In doing so, engineers have found a way to push advanced systems down to users quite quickly, given the size of the organization and bureaucracy involved.

Unfortunately, leaders receiving this technology have observed issues with usability. Very few 
designers understand the challenging use cases experienced by soldiers in austere environments, and even fewer take the time to incorporate these considerations into a deliberate and holistic design of the user experience. Commercial systems designed for use in everyday life are being rudimentarily modified for use in unpredictable (field) situations and environments. On the whole, outside of the Army's doctrinal DOTMLPF system (Department of the Army, 2013), which [slowly, but deliberately] assesses systems for integration with Army property, training, and logistics, there is very little unbiased assessment being done on the usability of these more hastily fielded $\$ 100$ million dollar programs meant to aid the warfighter (Army Science Board, 2002).

This study intends to fill that void by assessing commercially available civilian technologysmartphones-for use in robotics control. Doing so requires an understanding of not only the use case, but also of the environment and special considerations for the over-tasked, burdened soldieroperator. Both the carefully documented user study (Section 4.5) and the controller interface serve as significant contributions to the body of work devoted to improving the user experience for military operators, as it pertains to robotic teleoperation.

\subsection{Smartphones in the Military}

Smartphones will find their way to battlefield, given their market-dominance and adaptable nature-a function of the unique combination of processor power, size, and high-resolution displays. One can envision a myriad uses for these devices: geo-location and situational awareness, logistics tracking, communication, and robotics. In fact, the U.S. military is already in the midst of arming soldiers with smartphones, although security and connectivity remain challenges to full-scale adoption. Tests at White Sands and Fort Bliss training areas have proven encouraging, putting 300 phones from multiple manufacturers through their paces (Milian, 2011). PEO Soldier, the proponent for Nett Warrior, anticipates issue to operational units later this year (McHale, 2014). While there are still unanswered questions regarding screen glare, ruggedization, and battery life, the Army, at least, is clearly committed to continuing development toward a standardized military smartphone and operating system.

Aside from its low weight, small size, and all-in-one nature, the primary advantage of smartphones as a battlefield multiplier are their inherent adaptability, as well as their familiarity (and therefore intuitive interface) to a young, and primarily male, user. For robotics applications, the device's screen is used for video display and operator feedback (battery, heading, etc.), paired with control. Traditional user control inputs are available via a limited number of (hard) tactile buttons plus any number of programmable soft, touchscreen buttons. Unfortunately, smartphones, specifically those with touchscreen technology, have seen limited adoption by the military due to real and perceived disadvantages when compared to traditional tactile systems: fragility, clumsy operation by gloved users, and a lack of physical feedback. Touchscreen controls are sensitive and require a great deal of visual attention, given their tactile deficiencies. That said, our intent is to provide an option for smartphone-based robot control using the device's only physical mode-tilt/rotation. By leveraging on-board accelerometers and gyroscopes, we will assess an attitude-aware smartphone controller better suited to meet the needs of dismounted troops using small, portable unmanned ground vehicles (UGVs) for reconnaissance and surveillance.

\subsection{Attitude-Aware Control}

This research includes development and testing to assess the suitability of an orientation-based, attitude-aware smartphone controller. By exploiting onboard micro-electromechanical sensors, proprioceptive device inputs (tilt and rotation) are used to overcome common user complaints regarding touchscreens in field environments, while independently assessing the usability of the designed interface. Attitude, in the context of this research, stems from the software object capturing the 
smartphone's three-dimensional pose with respect to its inertial frame (gravity). Smartphones are attitude- (or pose-) aware thanks to their accelerometers and gyroscopes, measuring linear motion and rotation respectively. By accessing the phone's knowledge of its orientation, applications can respond to custom gestures, recognize orientation changes, and monitor dynamic motion. This can improve the user experience by providing a proprioceptive mode of feedback, removing clutter from the display, and mapping to mental models more intuitive to operators. While not "tactile," per se, users do benefit from the sense of device orientation provided by feeling the device move in their hand(s) (e.g., degree of tilt or wrist pronation).

\subsection{Contributions}

The outcome of this research contributes the following products to the greater body of knowledge surrounding human-robot interaction and multi-modal control. While development of the attitudeaware control interface lies at the core of our work, many other contributions were made.

1. A well-defined use case describing a specific, anticipated military mission augmented by UGVs: reconnaissance and surveillance (Section 3.1).

2. An understanding of the current operator control systems, as well as how tilt-based controls have been implemented in entertainment and gaming. This provides the starting point for our own custom interface and identifies best practices and/or design pitfalls (Section 1.1 and Table 1).

3. Identification of key heuristics governing successful inclusion of attitude-based control-a useful byproduct of controller development, and one that can benefit many future designs intended to incorporate proprioceptive robot control (Section 3.5).

4. A comprehensive user study assessing usability of the aforementioned control interface, comparing it against a virtual (touchscreen) joystick (Sections 4-6).

The remainder of this paper presents the supporting literature, interface design, experiment protocols, and results, as outlined above.

\section{Related Literature}

Robotics, and specifically the human-robot interface, is a complex interdisciplinary field requiring a blend of psychology, engineering, and computer programming. This research relied heavily on each, leading to a brief survey of currently available OCUs and of related literature tying together teleoperation, tilt-based control modes, and human factors, and usability.

Robotic systems are customarily comprised of three components: the control interface, the communications link, and the robot (Winfield, 2000). Where possible, the robotic system developed for evaluation followed accepted heuristics for (tele-operated) ground vehicles. The control interface was designed to demonstrate best practices with regards to display and content, and experiment protocols built heavily off of previously reviewed studies paired with ISO standards.

\subsection{Teleoperation}

Currently, the military is primarily invested in robots that are tele-operated or "manually controlled by an operator at a distance that is too great for the operator to see what the robot is doing" (Carlson \& Murphy, 2005; Murphy, 2000). A human user is crucial to operations in unknown environments, where their flexibility and expertise are needed to interpret and make judgments based on remote feedback (Winfield, 2000; van Erp, 1999). Historically, military robots have used direct teleoperation interfaces supporting real-time decision making. These are often hand controls-like 3-axis 
joysticks-paired with video feedback available from a robot-mounted camera. Fong and Thorpe (2001) describe this as "inside-out" driving, because the operator feels as if he/she is inside the vehicle looking out. This tends to challenge tele-operators, because performance is "limited by the operator's motor skills and his ability to maintain situation awareness...difficulty building mental models of remote environments...[and] distance estimation and obstacle detection" (Fong, Thorpe, \& Baur, 2003; McGovern, 1987).

In 1999, Jan van Erp conducted a thorough survey of teleoperation and operator tasks with a specific interest in improving the user interface without taxing communications links. He discusses at length the human operator's superiority at obstacle avoidance at high speeds and expertise regarding camera control and scanning (i.e., knowing where to look); however, he notes the difficulty for operators in the sensory deprived state inherent to teleoperation (van Erp, 1999). To combat this sensory deprivation, van Erp suggests several heuristics guiding the design of UGV's and their image system:

1. The field size of normal drivers is $140^{\circ}$. Smaller fields of view limit peripheral vision and may cause drivers to initiate their control actions earlier than optimal (van Erp \& Padmos, 2003; J. Y. C. Chen, Haas, \& Barnes, 2007). van Erp recommends a minimum field size of $50^{\circ}$, with $100^{\circ}$,preferred.

2. While fields of view may be artificially expanded by applying a magnification factor less than 1.0, van Erp has proven this disastrous for driving performance as it alters the user's perception of speed and distance and transfers less object motion to the display.

3. van Erp quotes his own experimental results to show that the required camera update rate, while task dependent, ranges between 3 to $10 \mathrm{~Hz}$.

4. Color images are preferred in rough terrain, especially when the interface lacks stereoscopic depth cues and/or when image quality is degraded.

5. While a variable-view camera (e.g., pan-and-tilt camera implementation) provides the operator a larger periodic field of view, many operators have difficulty reconciling the viewing direction of the camera from the vehicle's heading. To combat such effects, van Erp recommends including adequate vehicle references within the camera field of view (e.g., some portion of the vehicle should be visible in the camera frame).

When good heuristics are not followed, human teleoperation performance issues arise. Most fall into two categories, those caused by remote perception (or lack of) (van Erp, 1999) or remote manipulation (J. Y. C. Chen et al., 2007). Remote manipulation is often studied as it pertains to network-based latencies and/or controller time lag. When system latencies exceed one second, users are forced to adopt a "move and wait" strategy, with variable lags being even more detrimental than fixed ones (Lane et al., 2002). While difficult to measure, network latency in our own system remained reliably below one second (the critical threshold) (J. Y. C. Chen et al., 2007).

\subsection{Tilt-Based Research}

The term "gestural," in the context of human-robot interaction, usually describes a vision-based interface system to track human gestures (Loper, Koenig, Chernova, Jones, \& Jenkins, 2009); however, gestures may also be measured and used by any device equipped with inertial measurement units (IMUs), such as Apple's iPhone, Nintendo's Wii, or even simple wrist-worn gadgets (Bland, 2008; Hinckley, Pierce, Sinclair, \& Horvitz, 2000; Pitman \& Cummings, n.d.; Yagoda \& Hill, 2011). So as not to confuse the reader, gestures specific to the latter category, using IMUs, will be referred to as attitude-aware or tilt controls throughout this body of work. 
Some of the earliest work examining tilt controls for small-screen handheld device interaction was inspired by the growing popularity of Personal Digital Assistants (PDAs) (Rekimoto, 1996). Motivated by the need to scale desktop applications for use on smaller systems, tilt-based inputs were developed for navigation of system menus, documents, and maps. Experiments indicated that users could manipulate onscreen menus fairly accurately with as little as $2^{\circ}$ of tilt, while normal operations occurred comfortably between $10-15^{\circ}$. This work quantifies the level of precision available to robotic tilt control implementations (down to $2^{\circ}$ ), reinforcing its feasibility for simple driving control (Rekimoto, 1996).

A more physiological approach to design of multimodal systems reported on the limits of human anatomy and the level of control possible via the wrist (Rahman, Gustafson, Irani, \& Subramanian, 2009). Three physical axes of rotation are documented: flexion/extension $\left(60 / 45^{\circ}\right)$, pronation/supination $\left(65 / 60^{\circ}\right)$, and ulnar/radial deviation $\left(15 / 30^{\circ}\right)$. A motion algorithm used to discretize the tilt-space relied upon a quadratic function where it was recommended that "the extremities of the range of motion are given the largest amount of space and less angular tilt is allocated to the middle of the range" (Rahman et al., 2009).

Several other researchers have published findings of studies using accelerometers and gyroscopes for tilt-based interactions (Jang \& Park, 2004; Oakley \& O'Modhrain, n.d.). One used PDAmounted accelerometers to recognize gestures, comparing the sensor's signal pattern to a "known" gesture pattern; this led to proposed methods used to recognize and filter both static and dynamic accelerations. A key to this approach is the identification of a threshold value, or programmatic deadzone, ensuring that user inputs are distinctly large enough to warrant robot manipulation. (Jang \& Park, 2004). Another notable lesson learned includes the need for a deadman switch, following Oakley's click-tilt-release interaction methodology (Oakley \& O'Modhrain, n.d.; Oakley \& Park, 2007). This ensures that tilt controls are not always active and aims to eliminate inadvertent control inputs. Likewise, in our research, it provides an opportunity to recalibrate sensors around the point of origin, correcting for IMU drift and ensuring more precise control over the full range of the device's motion.

\subsection{Human Factors/Usability}

"Usability comprises ease of use and usefulness, and these drive user satisfaction. User satisfaction, in turn, results in usage" (Israelski \& Lund, 2003; Ling, Hwong, \& Savendy, 2007). ISO 9241, Ergonomics of Human-System Interaction (ISO 9241, 2008; Jokela, Iivari, Matero, \& Karukka, 2003) focuses on effectiveness, efficiency, and satisfaction, providing a blueprint for assessing usability (Jokela et al., 2003). Shneiderman's text summarizes these into five usability measures (Shneiderman \& Plaisant, 2010):

1. Time to learn. How long does it take for a typical user to learn the system, specific to a set of tasks?

2. Speed of performance. How quickly can users carry out those tasks?

3. Rate of errors by users. How often do users make errors, and of what type?

4. Retention over time. How well do users retain knowledge gained about system operation? Hours? Days?

5. Subjective satisfaction. This is a measure of how well users like various aspects of an interface, often collected via interview or survey.

Convention dictates that user studies incorporate these metrics into data collection to help quantify usability. The first four are directly measurable, while the fifth relies upon survey instruments or other data gathering techniques. When human performance is of interest, which commonly includes mental workload, the NASA Task Load Index (TLX) (Donmez, Pina, \& Cummings, 2008; Hart 
\& Staveland, 1988) may be administered. The System Usability Scale is a likewise standardized survey primarily implemented for software evaluation, and it was given in our study to participants after trials with each level of the independent variable (Brooke, 1996).

\section{Application Development}

Attitude-aware controls use IMUs, such as accelerometers and gyroscopes, to measure linear motion and rotation to monitor device pose and changes to it. Our implementation of attitude-based control, designed for robot teleoperation, accepts attitude inputs resulting from user gestures and maps these movements to robot behavior (throttle and heading). The goal was not simply to produce a prototype application, but to identify the design heuristics and features that most greatly impacted usability.

\subsection{Controller Use Case}

To best understand the application and its behavior, it is important to first obtain a frame of reference regarding what the controller was designed to do. What follows is a casual use case, describing the goals, primary actors, and conditions for use, followed by a common scenario describing how the application might be employed (Cockburn, 1997a, 1997b).

3.1.1 Goal: The goal of users engaged with this controller may vary slightly based on mission, depending on the type of reconnaissance and surveillance required. On the whole, users hope to guide a small, mobile robot equipped with a moveable camera into dangerous or hard to reach places in order to gain information about the environment. Successful operations would provide the user with the intelligence he/she requires while operating from a safe location some distance away.

3.1.2 Actor: The primary actor (user) is anticipated to be a young, male dismounted soldier between 18-35 years of age. He may have some college education and grew up with technologycomputers, video games, and smartphones. His primary specialty in the Army is something besides robot operation, affording him limited time for formal hands-on teleoperation training (likely not exceeding 40 hours) (Knox, 2012; Morrow, 2012). Some of these users might relish the opportunity to use "cool" technology, while others will feel it is an undue burden "not in their job description."

3.1.3 Conditions: Realistic use conditions (uncertain field environments) are hard to describe; however, users are expected to be almost exclusively outdoors during robot operation. At best, the operator might be behind a wall or in a doorway. Lighting conditions may cause issues with screen glare (bright sunlight) or eye strain and fatigue (bright screen in the dark). The user will likely be stationary-standing, squatting, or lying down-with a team of fellow soldiers providing security while he devotes his attention to robot operation. Security considerations may limit the duration of operations, so speed is crucial.

All soldiers (and therefore all users) wear military utility gloves and eye protection (both tinted and clear) for tactical operations. The robot may be one of many ruggedized, tracked vehicles under 35 pounds and small enough to fit in the soldier's rucksack. The smartphone controller is protected by a waterproof case and can be carried in one of the soldier's cargo pockets.

A note on gloved control. Soft buttons are touchscreen buttons, reacting only to conductive inputs like those provided by a user's fingertip flesh. To use them, gloved users must either remove their gloves or use touchscreen-compatible gloves that interweave special fibers into the fingertips. Hard buttons are tactile push buttons and can generally be felt even in the presence of gloves. The iPhone has four tactile buttons: the power button, the home button, and the volume up and down buttons. One option to accommodate gloved users may require elimination of most touchscreenbased interactions by utilizing the volume up and down buttons, located on the top edge of the phone, when positioned for control in landscape mode. 
3.1.4 Operation To drive the robot, the user presses and holds a deadman switch (touchscreen or volume button) to activate the controls, and then moves the device like a steering wheel. Tilting forward and back controls the robot's speed, while rotation left and right controls heading. When the user wants (or needs) to stop the robot, he simply removes his finger from the deadman switch and the robot stops (but does not brake).

To scan the robot's surroundings, the camera deadman switch is depressed and the device is again moved like a steering wheel, this time to pan ("steer") the camera left and right or tilt up and down. To stop the camera at any position, the deadman switch is simply released. To capture a photograph from the robot's camera, the user taps a snapshot button, visible only when camera controls are active. When the picture saves to the device, an alert view pops up indicating success. The user hits OK to exit and return to robot control; use of this alert is intended to reassure the user that sensitive information has been captured but can be suppressed based on mission needs.

\subsection{User Interface}

The graphical interface presented to the user was designed to be intuitive and accessible, regardless of technological proficiency or previous system experience. Low-level sensor inputs and controls were mostly hidden from the user and presented in forms easy to understand (e.g., battery icon versus remaining voltage). The motion input portion of the controller required users to hold and move the device like a steering wheel, using a mental model most would find familiar. This control interface prioritized display of the robot's camera feed and limited the clutter of other forms of feedback, such as connection, battery, and driving status.

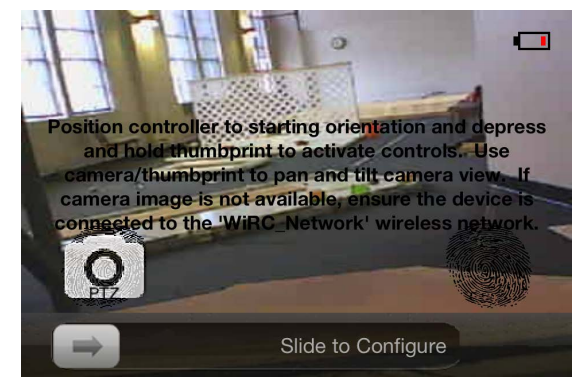

(a) Controller Home Screen

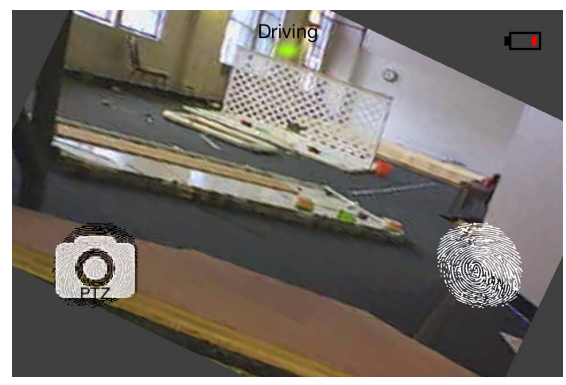

(b) Driving With Controller

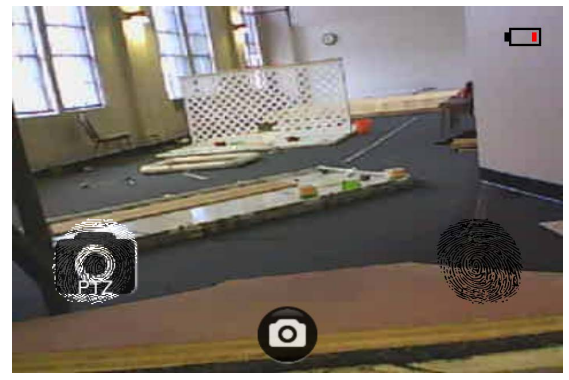

(c) Controlling Camera

Figure 2. Views of Controller Application

Fig. 2a shows the "home screen" of the attitude-aware controller used in most phases of this study. The video feedback takes up the entire screen in landscape mode ( $320 \times 480$ pixels), with soft-button thumbprints (i.e., deadman switches), overlaid on the left and right indicating where 
the user should press and hold to activate the attitude sensors for either the camera (left) or the vehicle motors (right). Along the top of the display (from left to right) is the controller connection status, the driving status, and the battery status. Driving status was comprised of three features: 1) a driving light that displayed green for driving, red for stopped, and blinking red for reverse; 2) driving text that read either "Driving" or "Reversing"; and 3) a visual representation of controller rotation, provided by keeping the video frame level to the horizon, similar to many aircraft attitude indicators (see Fig. 2b).

A brief set of instructions displays onscreen until controls are activated for the first time. The slider along the bottom of the screen allows access to the settings menu-a feature only made available in later phases of this research. Finally, the camera snapshot button in the bottom center of the screen allows users to capture a still photograph via the vehicle's onboard camera (see Fig. 2c); these save automatically to the phone's camera roll.

\subsection{Development Environment}

The Apple iPhone and iPod Touch ${ }^{\circledR}$ were selected as controller hardware for this project due to their well-supported development environment, XCode, and their standardized device characteristics: processors, operating system, and micro-electromechanical systems (MEMs). These devices run Apple's iOS, which is coded in Objective-C, an object-oriented programming language (Conway \& Hillegass, 2010; Hillegass, 2011). The iDevices used in this research are equipped with 3-axis accelerometers and gyroscopes, which detect and measure motion and rotation about three axes: $x$, $y$, and $z$. Primarily, they contribute to the user experience by detecting orientation changes but can be tailored for use in almost any instance where motion-based inputs are recorded (e.g., pedometers, panoramic picture applications, and tilt-based games). Apple makes this possible through their Core Motion framework, encapsulating accelerometer, gyroscope, and magnetometer data in multiple forms. Developers can choose to access any of this sensor data independently or as attitude, where sensor data are fused into a representative output. This output can be represented mathematically as Euler angles, a rotation matrix, or as a quaternion, all properties of the attitude object (Apple, 2011). Using a device motion manager, developers define the device reference frame, the type of attitude output requested, and the frequency with which such data should be captured.

\subsection{Motion Algorithm}

As arguably the most important part of this application's development, substantial time and effort was devoted to writing a motion algorithm capable of reliably handling complex three-dimensional motion. A survey of available tilt-based games and applications for the iPhone was conducted to collect and examine ideas and implementations that could inform this design (see Table 1). The majority of these games, built primarily for entertainment purposes, did not stand up to rigorous testing under use case conditions. Generally controls dictated a single user starting position, where a prescribed neutral point had to be found and held in 3D space. Almost none accounted for users who would be operating in unconventional positions (e.g., lying down), and those that did accounted only for "inversion," a specific scenario where the device's screen is pointed down, toward gravity, and accelerometer values are therefore reversed. Apple provides no specific guidance on how to use accelerometer and gyroscope data, leaving many researchers to struggle with implementation. Most have not completely solved the problem of how to orient and calibrate the system due to the variance in user's mental models affecting how each expects the device to behave. Researchers at the Massachusetts Institute of Technology designed a tilt-based controller for micro-air vehicles where users were observed inadvertently flying the aircraft backward, given their instinct to angle the device for comfortable viewing. This differed from the designed neutral angle (level to the ground), leading to the inadvertent (and undesired) behavior (Pitman, 2010; Pitman \& Cummings, 
n.d.). Similar problems manifested in early versions of our own attitude-aware controller, motivating research to conquer this obstacle to intuitive control.

Therefore, the centerpiece of our controller application software is really the robust motion algorithm developed to reliably handle complex three-dimensional motion while providing the flexibility to adapt to user's changing conditions/desires. Quaternions-a fourth-dimensional extension of complex numbers-were key to facilitating this, greatly simplifying the motion algorithms while tackling common issues that affect human-robot interaction, namely, starting orientation and gimbal lock. Further details on this algorithm and the quaternion-based approach can be found in Walker (2013).

\section{Table 1: Survey of Tilt-Based Games and Products}

\begin{tabular}{|c|c|c|c|}
\hline Product & Developer & Key Features & Reference \\
\hline $\begin{array}{l}\text { AR. } \\
\text { Drone }\end{array}$ & Parrot & $\begin{array}{l}\text { A unique hobbyist quadcopter controlled via iPhone, and a development kit for more advanced } \\
\text { controls and gaming is open source. The packaged controller was recently updated to v2.0 and } \\
\text { now supports both relative and absolute control modes. In the relative mode, the aircraft is the } \\
\text { point of reference, and all tilts are translated into movement with respect to it. In absolute control } \\
\text { mode, the pilot is the point of reference, and all tilts are translated into movement with respect } \\
\text { to him or her. It is meant to provide a more intuitive interface for first-time pilots, or those } \\
\text { who lack experience with remote control platforms. Absolute mode almost assures that users } \\
\text { are co-located with the quadcopter, as true teleoperation would work best in the older, relative } \\
\text { control mode. This controller does use attitude in two dimensions; however, it does not account } \\
\text { for controller inversion. It uses separate accelerometer and gyro streams rather than fused sensor } \\
\text { data, leading to gimbal lock. }\end{array}$ & $\begin{array}{l}\text { (Yarrish, } \\
\text { 2011; } \\
\text { Piskorski, } \\
\text { Brulez, } \\
\& \text { Eline, } \\
2011 \text { ) }\end{array}$ \\
\hline $\begin{array}{l}\text { WiFi } \\
\text { RC }\end{array}$ & Dension & $\begin{array}{l}\text { This application was developed to work out of the box with the WiRC hardware described in } \\
\text { Section } 4.3 \text { for operating remote control vehicles. Its tilt mode requires the user to pick which } \\
\text { gyro will control which channel (non-obvious in landscape mode). It then provides feedback } \\
\text { regarding angle of input via small tick marks that move along the perimeter of the screen. By } \\
\text { pressing the "play" button, users can recalibrate the tilt control's center point; however, calibration } \\
\text { does not correct for inverted use. There is no deadman switch, meaning controls are always } \\
\text { active; presumably a user could stop by pressing the play button and re-setting the controller to } \\
\text { zero. Gimbal lock occurs when the device is level to the ground. }\end{array}$ & $\begin{array}{l}\text { (Dension, } \\
\text { 2011) }\end{array}$ \\
\hline $\begin{array}{l}\text { Tilt to } \\
\text { Live }\end{array}$ & Alex Okafor & $\begin{array}{l}\text { In this game, the objective is for the user to maneuver his or her arrow around obstacles on the } \\
\text { screen. The user interface provides options for orientation (choose from regular }\left[45^{\circ}\right] \text {, top-down } \\
\text { [level with ground, or custom) as well as tilt sensitivity, adjusted separately for each axis. The } \\
\text { custom orientation allows a user to set a neutral point and does account for inverted reference } \\
\text { frames. Users are prompted to choose an orientation before the start of each new game, and the } \\
\text { interface is intuitive enough for even new users to understand, using graphics to demonstrate the } \\
\text { choices. }\end{array}$ & $\begin{array}{l}\text { (Okafor, } \\
\text { 2010) }\end{array}$ \\
\hline $\begin{array}{l}\text { Mad } \\
\text { Bomber }\end{array}$ & $\begin{array}{l}\text { Charles Sca- } \\
\text { lesse }\end{array}$ & $\begin{array}{l}\text { The author provides details of his motion algorithm in the reference provided. This game requires } \\
\text { the user to tilt to move their character onscreen in an effort to catch dropping bombs. Uniquely, } \\
\text { this game provides accelerometer calibration that supports any device play orientation, including } \\
\text { inversion. This is accessed via an options menu that prompts the user to orient the device at the } \\
\text { desired angle and then press a button to calibrate. Inversion is handled separately. Controls do } \\
\text { not seem to be properly filtered to account for sensor drift, and only one-axis motion is measured, } \\
\text { making the algorithm less complex. }\end{array}$ & $\begin{array}{l}\text { (Scalesse, } \\
2013)\end{array}$ \\
\hline Sphero & Orbotix & $\begin{array}{l}\text { This application is used to control a small, robotic ball. The tilt-based controls (available in } \\
\text { addition to joystick controls) are fairly elementary. They do not account for user-defined origins, } \\
\text { meaning users must start with the device parallel to the ground. It likewise does not operate } \\
\text { correctly inverted. There is no deadman switch operation, making it much too easy to lose control. }\end{array}$ & $\begin{array}{l}\text { (Orbotix, } \\
\text { 2012) }\end{array}$ \\
\hline $\begin{array}{l}\text { Cube } \\
\text { Runner }\end{array}$ & Andy Qua & $\begin{array}{l}\text { This game, similar to Tilt to Live, has users represented as small flying arrows through a three- } \\
\text { dimensional landscape filled with cubes that they must navigate around. It is presented in portrait } \\
\text { or landscape mode (i.e., the only one surveyed) and tilting OR rotating left and right steers the } \\
\text { aircraft. Tilt does not control animation speed. In the settings, users can calibrate the center } \\
\text { point from which movements are measured along the single axis utilized. In testing, calibration } \\
\text { successfully overcame starting positions where the device was purposefully off-center by up to } \\
30^{\circ} \text {. Additionally, it automatically detects cases of inversion, even during active game play, and } \\
\text { makes behind-the-scenes adjustments to ensure controls are not reversed; however, it does not } \\
\text { account for users who wish to leave the device parallel to the ground (i.e., resting on a table), as } \\
\text { gimbal lock prevents measurable rotation at this attitude. }\end{array}$ & $\begin{array}{l}\text { (Qua, } \\
\text { n.d.) }\end{array}$ \\
\hline
\end{tabular}


Walker et al., User-Centered Design of an Attitude-Aware Controller for Ground Reconnaissance Robots

\subsection{Heuristics for Tilt-Based Control}

As previously stated, the goal of this research was not simply to produce a prototype application, but to identify the design heuristics and features that most greatly impacted usability. This was done throughout an iterative development process, using several pilot tests to identify best practices. Heuristics developed from the features (and their settings) that systematically produced the best results and most satisfying user experience.

While the aforementioned controller application was designed and developed for Apple smartphones, the heuristics governing its implementation may be applied to any handheld device using a similar suite of attitude-aware sensors employed for robotics control. A few of these design guidelines are very specific to the motion algorithm, which is beyond the scope of this publication. Others are more generic regarding basic controller behavior, as enumerated below.

1. All device motion should be measured relative to the point where the deadman switch was activated. This ensures that users do not have to expend mental effort finding the device "origin"; instead, the device resets itself at each control activation.

2. Given that the bulk of control is done via gestural manipulation of the device body, and all robot manipulation is done via tele-presence, the controller screen should be almost wholly devoted to video feedback and therefore in landscape mode.

3. Driving and camera controls should not be activated simultaneously. Users must choose whether to drive the robot or manipulate its camera at any given time. Enforcing this limitation simplifies the control task for novice users, which most soldier-operators are, and avoids disorientation in individuals new to teleoperation.

4. All feedback is provided to the user via video from the robot's camera, meaning that the camera should remain in a fixed (trimmed) position while driving. This camera-neutral position is the same for all users. Each individual sees the same frame with respect to the robot to ensure best practices are maintained (e.g., keeping some portion of the robot chassis in view).

5. Tilt-based camera inputs are best implemented when the user imagines that the robot's camera is attached to the controller itself. Like taking a panoramic picture with the smartphone, users can sweep the controller broadly from left to right to pan the robot's camera and scan its surroundings. It is almost as if operators are using the control device to select a viewing frame of a broader window.

6. The camera, when stopped, should remain in its current position. This gives the user an opportunity to fix the camera in a given location for reconnaissance, for closer examination of an object, or when capturing a photograph.

7. When the camera is at a position other than neutral, and the user depresses the driving deadman switch, the camera shall immediately snap back to its neutral position and remain there until once again manipulated. This prevents issues with users not being able to resolve the camera's position from the robot's heading.

8. Given the limitations of a wireless network, periodic interruptions to the connection may occur. If the controller disconnects from the robot, the robot should automatically stop. A popup then notifies the user of the disconnection and prompts reconnection by hitting a button. Doing so re-establishes the connection with the robot and should permit the user to continue operation after only minimal disruption. 


\section{Method}

Following a robust development phase, the basis of this research is to identify and assess an operator control unit methodology using tilt-based modes that promote simple, efficient control of small reconnaissance robots via smartphone. If specific usability issues are addressed, these devices have the potential to replace a number of proprietary, platform-specific controllers currently in use. To test this assertion, and the controller application prototype, a formal usability assessment was conducted with human users. This required a multi-phase experiment to help answer the question, "Can teleoperated control of ground robots be implemented using smartphones such that their advantages (performance, usability, and size/weight) overcome suspected deficiencies, including negative user perception?" Several hypotheses were developed and tested using the experiment protocol that follows.

\subsection{Related Work}

The Army Research Laboratory (ARL) has done introductory work with smartphone-based controllers, including a series of scalability experiments began in 2008 (Redden, Elliott, Pettitt, \& Carstens, 2011). In the second of the series, Pettitt, Redden, Fung, Carstens, and Baran (2011) and Fung (2011) describe an experiment where an Android-based virtual joystick controller (see Fig. 3) is programmed to provide the same functions as a gaming controller (XBox360) while combining the display and control functions in one space. The XBox360 controller was chosen as the baseline for comparison due to its fielding with the iRobot PackBot and its use in earlier experiment trials related to ARL's scalability research.

Participants used each controller to drive a PackBot Explorer robot on two courses-indoor and out-after a brief training period. Most maneuvering was conducted beyond line of sight of the operator, requiring video feedback be provided on the Android phone in all instances. User performance was measured via time to complete the course, number of driving errors (collisions), and number of course errors (driving outside the marked areas). Participants, all military service members, also provided feedback via the NASA Task Load Index (TLX) and surveys designed to assess controller feasibility and usability.

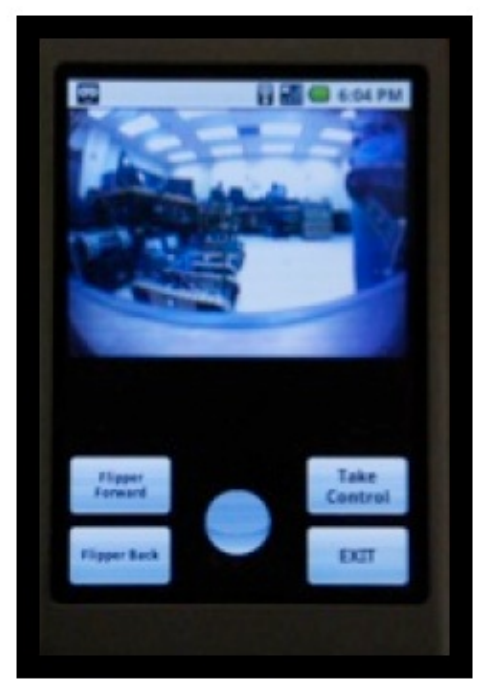

Figure 3. Army Research Laboratory’s Android Operator Control Unit (Pettitt et al., 2011) 
Users generally performed poorly with the virtual joystick, with three variables reaching significance $(p<0.001)$ : mean time to complete the courses, mean number of off-course errors, and mean number of driving errors. Additionally, participants averaged a higher total workload score with the Android controller, specifically on the mental, effort, and frustration scales. Finally, users assessed their own performance with the virtual joystick controller poorly, with 26 of 30 participants preferring the traditional control option (Pettitt, Redden, Fung, Carstens, \& Baran, 2011). While most users appreciated the Android's light weight, small size, ease of use, and one-handed operation, many complained about the lack of physical feedback and sensitivity of a rather small virtual joystick. The authors suggested providing larger buttons, larger spacing between buttons, and incorporating some type of haptic or audio feedback, as many users experienced difficulty identifying when the virtual joystick was "engaged."

Picking up where this left off, our multi-phase experiment protocol expanded on this study by introducing a second smartphone-based option (attitude-aware controls) designed to overcome many of the user-perceived deficiencies of ARL's virtual joystick. By closely mimicking the experimental design of Pettitt et. al. (2011), this usability experiment began by comparing ARL's version of virtual joystick control to the newly designed attitude-aware controller; our goal being to determine whether the improved design characteristics of the tilt-based controls translate to a suitable, more satisfying smartphone-based control option.

\subsection{Participants}

Twenty-five participants were recruited primarily from the undergraduate and graduate engineering populations at the University of Oklahoma. ${ }^{1}$ They ranged in age from 18 to 51, with a median age of 26. The subject population consisted of 21 men and 4 women, recruited to represent the military's gender bias (approximately $85 \%$ male and 15\% female). While four participants reported military experience, none were exposed to unmanned systems during their service. Only one individual reported left-hand dominance.

All participants were compensated a total of $\$ 40$ for five-hours of experimentation over six weeks. No previous experience with either smartphones or robots was necessary to participate, although $76 \%$ reported good or excellent proficiency with mobile devices, with 22 respondents reporting at least 12 hours of weekly use. Questions regarding use of remote controlled systems of varying intelligence indicated that nine participants had little to no experience with ground vehicles, and only one reported excellent proficiency. More participants reported exposure to systems using gestural based inputs, the most common being Nintendo's Wii; 20 participants reported experience with that system, and 14 participants had played tilt-based games on a mobile device, such as the iPhone. Finally, a spatial reasoning test (University of Kent Careers and Employability Service, 2013) was administered to assess users' natural spatial abilities, a proven predictor of user performance in teleoperation tasks (T. Chen, Yesilada, \& Harper, 2010; Tomlinson, 2009; Tracey \& Lathan, 2001). The mean score for study participants was 13.4 out of 20 , with a median of 14 , matching the referenced average for all test takers. Only two subjects tested notably below average, with scores of 8 and 9 .

\subsection{Apparatus}

Experiment trials took place using a modified Kyosho Blizzard SR Ready-to-Run (RTR) remote control tracked vehicle (Fig. 4a). The factory-provided radio frequency (RF) module was swapped for a Dension WiRC WiFi over RC system², which accommodates eight analog input channels plus

\footnotetext{
${ }^{1}$ Permission to proceed with this study was granted by the University's Institutional Review Board (IRB) on 31 August 2012, filed under IRB \#1115. All necessary disclosures and forms specific to that process are on file with the institution.

${ }^{2}$ System comes with everything shown in Fig. $4 \mathrm{~b}$
} 
two USB video streams and communicates via WiFi dongle over standard $802.11 \mathrm{n} / \mathrm{g} / \mathrm{b}$ wireless in the $2.4 \mathrm{GHz}$ range. Other vehicle modifications included the addition of pan-tilt Lynxmotion camera mounts for the Logitech C110 USB camera and two standard servos to power their motion (see Fig. 4c). This setup provided approximately $120^{\circ}$ of motion to pan left and right or scan up and down. The fixed camera driving position looked down the centerline of the robot with its front "bumper" in view along the bottom of the frame, coinciding with studies that indicate video feedback should provide a visual reference to the device chassis (Keyes \& Yanco, 2006; Wang, Lewis, \& Hughes, 2004). Overall, while smaller and less rugged than the PackBot used in ARL's studies, the Kyosho Blizzard remains a highly maneuverable tracked robot providing a reasonable, if less refined, platform for comparison.

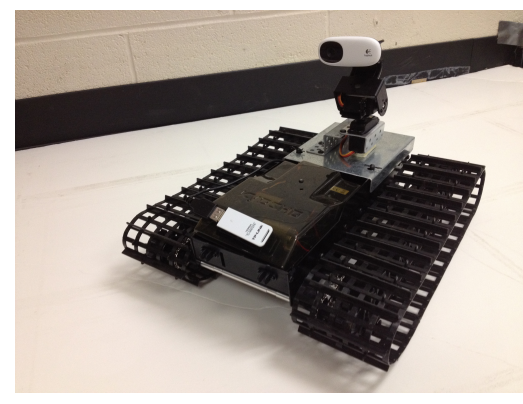

(a) Kyosho Blizzard SR RTR Modified for Teleoperation

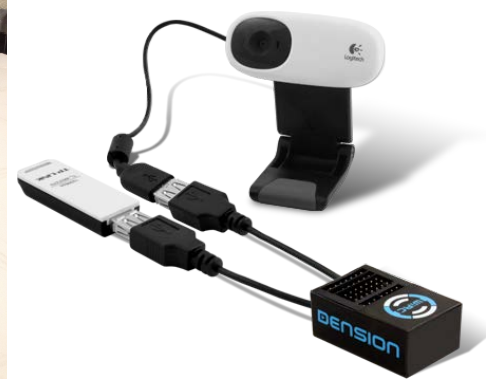

(b) Dension WiRC WiFi Over RC (Dension, 2011)

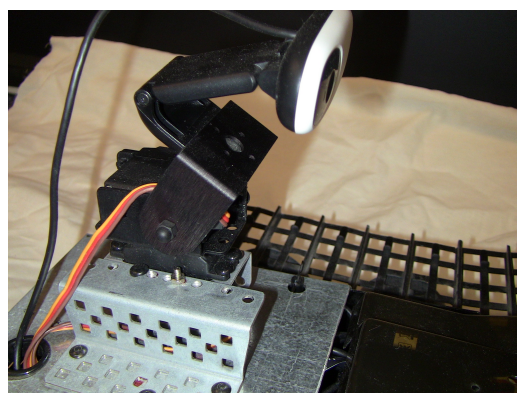

(c) Lynxmotion Pan/Tilt Camera Mount With Servos

Figure 4. Experimental Hardware

Control of the robot was achieved via custom application, introduced briefly in Section 3 and developed for Apple's iOS and associated devices, was used throughout this experiment. An indoor robotic test course was designed to test users on both mobility and reconnaissance tasks. Rooms were setup to mimic an urban business or residence with obstacles littered throughout the course. These varied in type: office furniture, bins, doorjambs, and other miscellaneous items. Doorways and corridors varied from larger 36" openings to narrower 22" openings; furthermore, the course included turns in both directions, including some that required maneuvering at more than $90^{\circ}$. See Fig. 5 for a schematic of the course layout and Fig. 6 for pictures of the setup.

In order to separate the effects of a user's ability to navigate (a measure of spatial awareness) versus their ability to maneuver (a function of the control effectiveness), a path through the course 


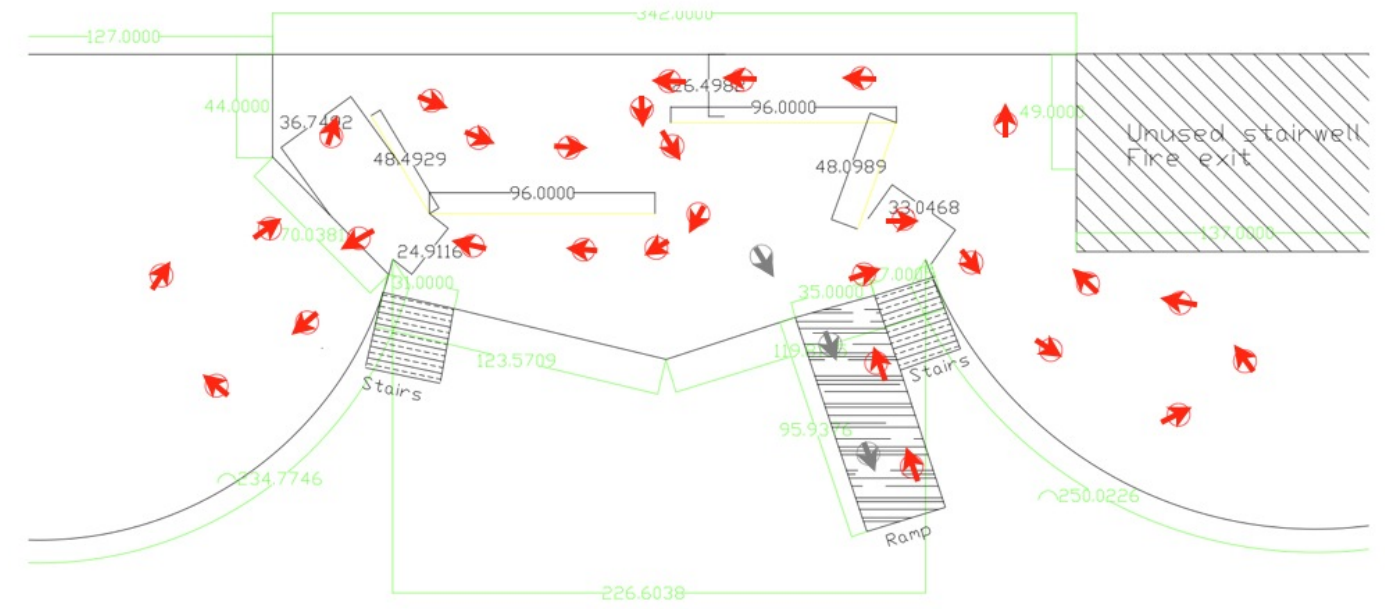

Figure 5. Layout of Test Course. Users began at the bottom of the ramp, travelled up and immediately to the right, following the red arrows until coming back down the ramp (then following the silver arrows).

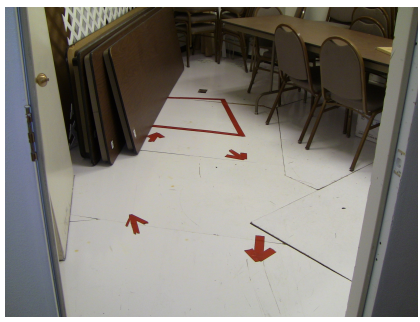

(a) Picture of Course (Left)

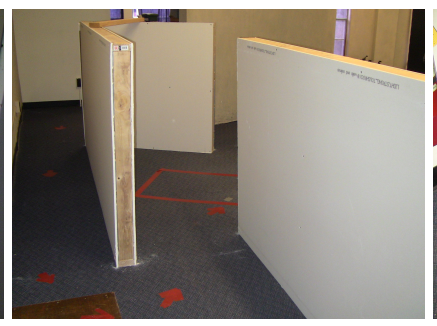

(b) Picture of Course (Middle)

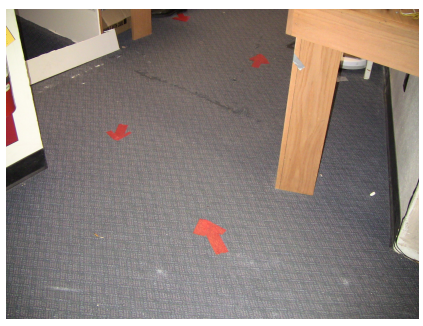

(c) Picture of Course (Right)

Figure 6. Pictures of Course

was marked with red duct tape arrows (depicted in the graphic) spaced 18-24" apart. These provided navigational cues, as users followed them counter-clockwise through the course, and they served as a measure of driving accuracy.

\subsection{Design}

Each experiment phase compared two similar but different controllers, using the tracked robot described in Section 4.3. Controller type was examined as a within-subject variable, given each user tested with both available controller options in each phase. The order in which independent variables were presented to users was counterbalanced for learning and ordering effects.

The independent variables in this experiment were controller type and task. Tasks remained constant within a phase, but changed between them, building upon a user's experience with the system to increase robot capabilities alongside task complexity. A total of five controllers were presented to each user over the course of three experiment phases. The third phase tested customization of user settings, such as controller sensitivity, responsiveness, and mode (i.e., joystick or tilt for each robot channel). Results indicate a lack of correlation between satisfaction and user performance but 
is outside the scope of this discussion. See Walker (2013) for further details and analysis. Phases 1 and 2 will be discussed here, as follows.

\subsection{Procedure}

4.5.1 Phase 1: Phase 1 was designed as a direct extension of Pettitt's ARL study. As such, an iOS version of their Android OCU was introduced, defined as Controller A (see Fig. 7a). Presented in portrait mode, the upper half of the screen provided video feedback, while the bottom half housed the virtual joystick. The joystick could be dragged freely in any direction; when released and/or returned to center, all robot motion ceased. While a capable controller, efforts were not made to "optimize" its control, rather only to sufficiently mimic that used in the 2011 study, in providing an accurate baseline for comparison. Controller $\mathbf{B}$ consisted of a driving-only attitude-aware control interface presented in landscape mode with full-screen video feedback. A thumbprint button located in the bottom right of the screen served as the deadman switch and activated tilt controls when pressed and held (see Fig. 7b). Each activation also re-leveled the controls, meaning that the attitude at which the deadman switch was depressed became the controller's new neutral point. Users then tilted the device forward and backward to control the throttle and rotated left and right to control heading. Releasing the deadman switch stopped the robot. The task included driving the robot around the course as prescribed during training. Users would be unable to see the robot, although they remained within auditory feedback range.

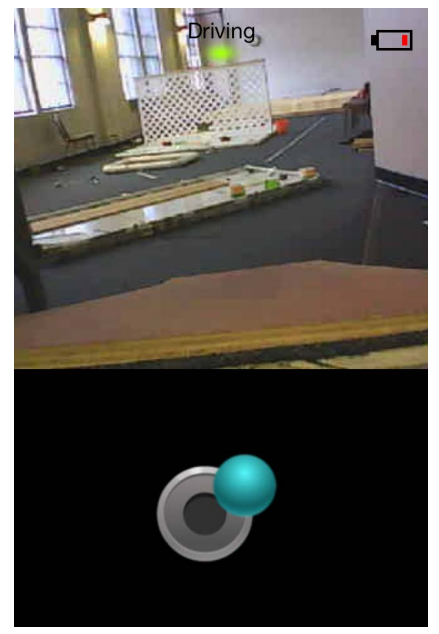

(a) Controller A: Joystick v1

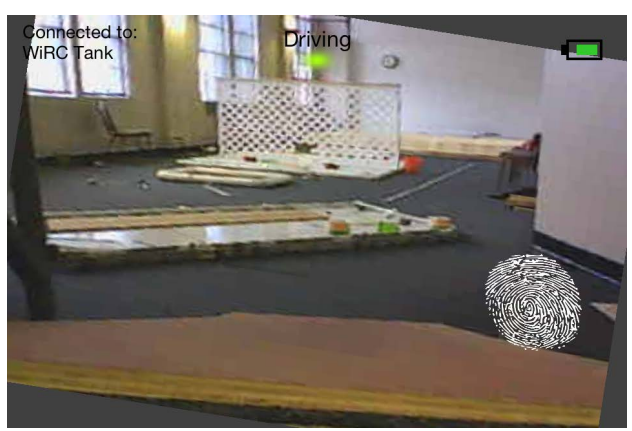

(b) Controller B: Tilt v1

Figure 7. Phase 1 Controllers. Task (and therefore control) included driving only.

4.5.2 Phase 2: Phase 2 gave users control of the pan/tilt camera affixed to the robot. In an effort to manage task complexity, camera motion could only be directed while the robot was stopped. In this phase, users were instructed to drive their robot like in Phase 1 and stop periodically to scan for objects along the way. To mitigate the effects of individual scanning strategy, duct tape boxes were added to the course (one in each room), from which users were directed to scan for the object(s) specified. Boxes were positioned such that object(s) would be visible from within them assuming users could properly scan all $360^{\circ}$ of a room. The objects used were colored foam balls, $3.5^{\prime \prime}$ in diameter. Users were presented the balls in advance of their timed runs and were informed of which color should be present in each room, though objects were not in position during training. Upon 


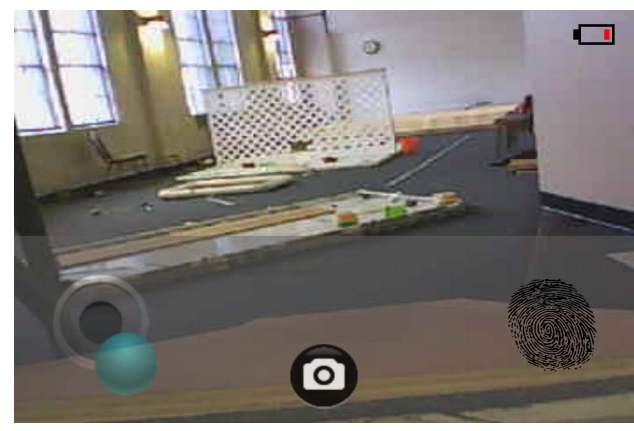

(a) Controller C: Tilt + Joy v2

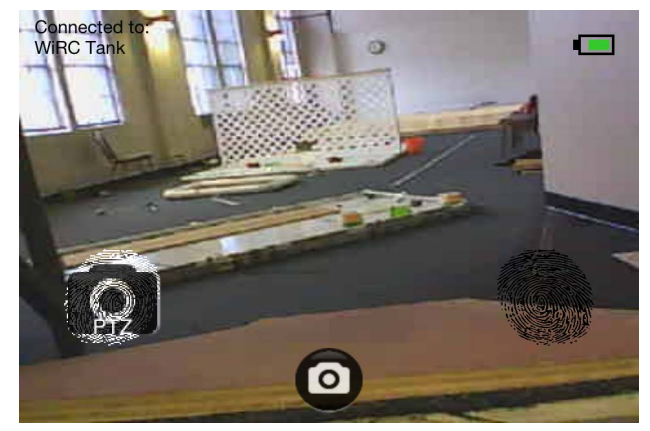

(b) Controller D: Tilt v2

Figure 8. Phase 2 Controllers. Task included driving and scanning for objects in each of the three rooms along the course. Users controlled the motors (maneuver) via tilt and tested both joystick and attitude-aware controls for camera pan/tilt.

finding an object, participants took a picture of it using the camera button at the base of the controller and mapped its approximate location on a worksheet provided. Users were asked to place a mark where they thought they had found the object. While not imperative to understanding the control task, these results were used as a measure of a participant's ability to localize their robot in space and plays toward overall spatial ability as it relates to teleoperation. Two runs were executed, $\mathrm{X}$ and Y. Each run consisted of a set of three object locations, one in each room. The runs were designed such that difficulty was roughly equal and objects could be found when the room was scanned but would not be found by accident in the course of driving. Users saw each run once, counterbalanced along with control type and order.

Both levels of the independent variable in Phase 2 used attitude-aware driving controls; however, implemented different modes (joystick and tilt) for camera manipulation. Controller $\mathbf{C}$ used a virtual joystick to control the camera pan/tilt. The joystick was roughly equal in size to the driving deadman switch and located in the lower left-hand corner (see Fig. 8a). Controller D presented the user with the driving deadman switch (a plain thumbprint button) on the bottom right of the screen, and a camera deadman switch (a thumbprint with camera overlay) on the bottom left of the screen (see Fig. 8b). It used tilt-based inputs for driving and camera manipulation. In both controllers, C and $\mathrm{D}$, the camera remained focused on its current target when the joystick or deadman switch were released, allowing users to look more closely at the frame and/or take a picture. To ensure users did not become disoriented when driving, the camera returned to its neutral position (facing forward on the centerline of the robot with the front bumper visible in the bottom of the frame) each time the driving deadman switch was activated (i.e., implicit mode switching).

The intent of adding the reconnaissance tasks to Phase 2 was to test the suitability of each type (mode) of control input for the robot's third and fourth degree of freedom (camera pan and tilt), while also examining the effects of interface mode confusion. Work by Chong and Lankenau indicates that mode confusion may result when users' mental models differ from the system's actual model or when feedback is insufficient to indicate operation mode (Chong, 2000; Lankenau, 2001). In Controllers $\mathrm{C}$ and $\mathrm{D}$, feedback is provided primarily via soft buttons highlighting upon touch, as well as the presence (or absence) of driving lights. Given the similarities of the control inputs (i.e., press, hold, and tilt), it was important to consider whether users experienced mode confusion with either set of controls, especially considering all robot degrees of freedom are accessed from the same interface screen. 


\subsection{Measures}

Experiment dependent variables consisted of both quantitative and qualitative information collected during the course of training and timed trials. Performance measures included practice time, time to complete the course (in seconds), number of major driving errors, number of minor driving errors, and number of path points hit. Driving errors were differentiated based on severity. A major error included instances where the observer had to step in and make a correction (e.g., repositioning the robot, putting it back on the course after a major deviation, etc.). While rare, experimenters felt it necessary to limit the time users spent in an unrecoverable position, so as to preserve the test data for comparison. Minor errors were collisions from which the operator could self-correct, often engaging reverse. The arrows depicting the path through the course were called path points and were considered "hit" if any part of the robot came in physical contact with them while traveling in the same direction that they were pointing. There were 32 total path points, and the individual administering the experiment noted the total number hit along with their location.

Following each timed trial, users were asked to complete the NASA TLX workload index and provide usability feedback via custom surveys hosted on SurveyMonkey.com. The NASA TLX was administered using a desktop application by Playgraph and included both the Likert scale and pairwise comparisons (Hart \& Staveland, 1988; Human Performance Research Group, NASA, 1988). Surveys were customized for each phase of the experiment and were vaguely inspired by the surveys used in ARL's study (Pettitt et al., 2011). In Phases 1 and 2, users completed post-iteration surveys after each timed trial and a post-experiment survey at the end of the phase. Survey questions focused on usability and user preference; most questions were answered on a five-point Likert scale, but some permitted free text commenting, providing perhaps the most revealing evidence of user attitudes.

\section{Results \& Discussion - Phase 1}

In Phase 1, the independent variable was controller type-A (joystick) or B (tilt-based)-making it a one-factor experiment with two levels. The 14 dependent variables used for statistical analysis were a combination of performance results, formal measures of workload and usability (NASA TLX and System Usability Scale), and informal measures of user satisfaction collected via survey. These metrics were collected for each user trial: 25 users $\times 2$ controller levels $=50$ trials.

\subsection{Statistical Analysis}

To test the null hypothesis (that users would perform equally well with both controllers), the $F$ statistic was calculated for each of the 14 dependent variables defined. The critical value to denote significance was $p<0.05$. Table 2 shows the means, standard deviations, and statistics for each dependent variable. Those achieving significance are highlighted in yellow.

\subsection{Performance}

Five variables achieved significance at the $p<0.05$ level: ttime, minerror, move, obstacles, and usability, leading us to reject the null hypothesis, given that controller means are not equal. Trial time (ttime) favored the joystick controller, matching observations made during experiment trials. Joystick runs were significantly faster than those with tilt controls. This appears to be the result of a small virtual joystick with limited travel between center and bezel. Users struggled to find the joystick's "sweet spot," resulting in most operating at top speed, or close to it, for the duration of their runs. Many adapted to those conditions by driving in short bursts, although collisions and minor errors were significantly higher as a result $\left(\bar{x}_{A}=5.64\right.$ vs. $\bar{x}_{B}=4.16, F=4.29$ at $\left.p=0.049\right)$.

The three other variables resulting in significance all favoring the tilt controller resulted from user responses to the post-iteration questionnaires. Users' rated ability to "move in the correct direction" 
Walker et al., User-Centered Design of an Attitude-Aware Controller for Ground Reconnaissance Robots

Table 2: The F-Statistic: Phase 1

\begin{tabular}{|c|c|c|c|c|c|c|c|c|}
\hline & & ptime & ttime & majerror & minerror & pathpts & tly & sus \\
\hline \multirow{2}{*}{ Controller A } & mean $(\bar{x})$ & 687.72 & 305.88 & 0.28 & 5.64 & 28.6 & 55.31 & 64.9 \\
\hline & $s t d \operatorname{dev}\left(s_{d}\right)$ & 213.19 & 69.4 & 0.61 & 2.6 & 2.02 & 14.78 & 16.9 \\
\hline \multirow{2}{*}{ Controller B } & mean $(\bar{x})$ & 648.68 & 339.56 & 0.4 & 4.16 & 28.28 & 53.89 & 68.7 \\
\hline & $s t d \operatorname{dev}\left(s_{d}\right)$ & 245.01 & 100.34 & 0.76 & 2.75 & 2.67 & 20.09 & 17.08 \\
\hline \multirow{3}{*}{\multicolumn{2}{|c|}{$\frac{p(<0.05)}{F}$}} & 0.445 & 0.046 & 0.417 & 0.049 & 0.55 & 0.695 & 0.258 \\
\hline & & 0.6 & 4.41 & 0.68 & 4.29 & 0.36 & 0.16 & 1.34 \\
\hline & & move & obstacles & slow & med & hi & drive & usability \\
\hline \multirow{2}{*}{ Controller A } & mean $(\bar{x})$ & 2.44 & 2.52 & 3.48 & 2.625 & 1.72 & 2.96 & 3 \\
\hline & $\operatorname{std} \operatorname{dev}\left(s_{d}\right)$ & 0.8206 & 0.8226 & 1.005 & 0.7697 & 0.9798 & 0.8888 & 1.04 \\
\hline \multirow{2}{*}{ Controller B } & mean $(\bar{x})$ & 3.52 & 3.08 & 3.68 & 2.8 & 1.88 & 3.2 & 3.56 \\
\hline & $s t d \operatorname{dev}\left(s_{d}\right)$ & 0.9626 & 0.9539 & 1.1445 & 1.08 & 0.8327 & 1.041 & 1.08 \\
\hline & $p(<0.05)$ & 0.0001 & 0.0076 & 0.4091 & 0.5884 & 0.3563 & 0.282 & 0.045 \\
\hline & $F$ & 20.68 & 8.49 & 0.71 & 0.3 & 0.88 & 1.21 & 4.46 \\
\hline
\end{tabular}

was referred to as the move variable. Multiple individuals noted that the joystick controller failed to operate as expected; many found it difficult to drive straight ahead. In many instances, this drift resulted due to disconnect between where users thought their thumb (or finger) was in contact with the joystick and where it actually was. Given the fingertip size of the joystick [ 75 x 75 pixels], a small variance in touch location yielded a noticeable effect on robot behavior. This phenomenon is believed to be a contributing factor to the difference in user ratings, leading to the significance observed in the move variable. Later control iterations (in Phase 3) would allow for control of the joystick size, slightly improving both user satisfaction and performance.

Users also rated the attitude-aware controller significantly better with respect to their ability to avoid obstacles, indicating that obstacle avoidance is a function of not only general teleoperation skills, but also related to the control type (or the user's comfort with it) in use. It is not immediately clear why obstacle avoidance was superior with the tilt controls, as users did complain that turning was more difficult. Observations indicated that most problems when turning in place occurred when users accidentally engaged reverse. It was natural for users to tilt the controller back toward themselves while rotating the device, and if, in doing so, the device tilted beyond the set neutral point, the robot began to drive backward. That said, users with tilt controls did report better control over the entire range of the robot's throttle, as indicated in answers to survey questions regarding control at slow $(\bar{x}=3.68)$, med $(\bar{x}=2.8)$, and hi $(\bar{x}=1.88)$ speeds (see Fig. 9b). It could be this perceived sense of control over the entire operating range that had the largest impact on users' self-rated ability to avoid obstacles.

While the formal measure of usability, sus (the System Usability Scale), did not achieve significance, qualitative user-rated usability, collected during post-iteration surveys, did. Given that SUS was developed as a general measure of system usability (in an era of desktop-based systems), it may not provide the most accurate picture of controller usability. Lending confidence to the more informal measure of user-rated usability, on a scale of 1 to 5 , is the fact that both it $(\bar{x}=3.56, p=0.045)$ and user preference (A: 36\%, B: 64\%) indicated support for the same controller (tilt-based). When, at the conclusion of the experiment phase, participants were asked to choose which controller they preferred, 16 out of 25 chose Controller B (see Fig. 9a). 


\section{Controller Preference}

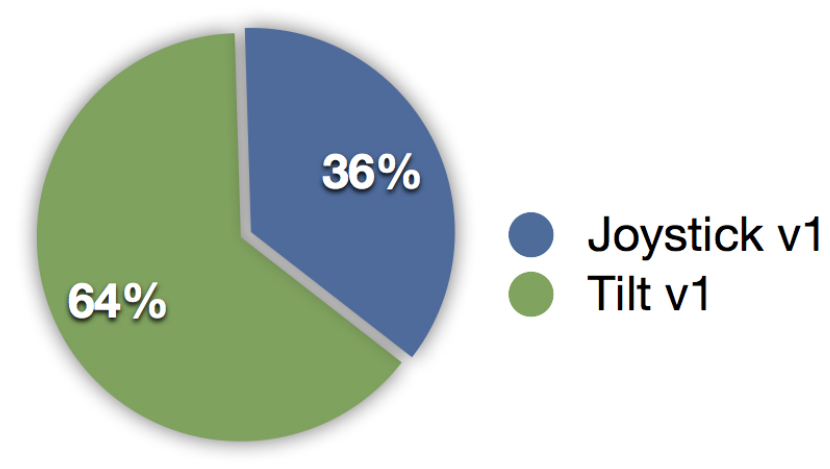

(a) Phase 1 Overall User Preference

(Virtual Joystick Control vs. Tilt Controls)

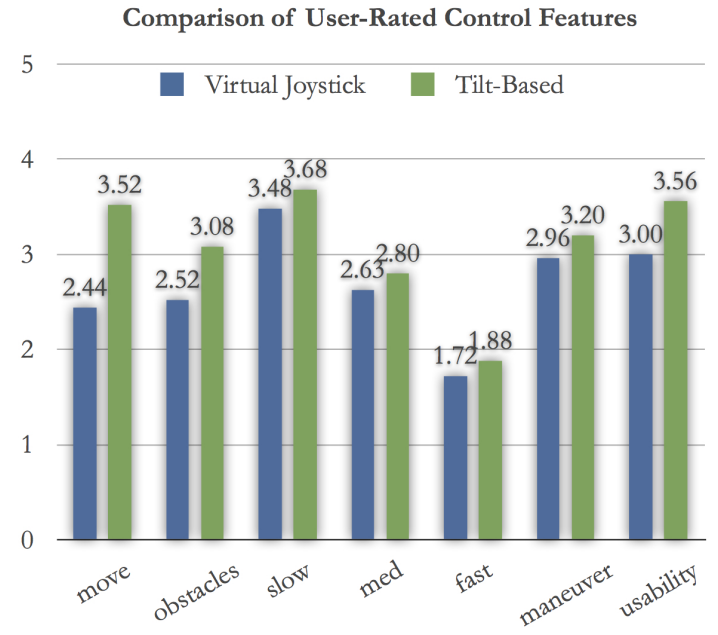

(b) Phase 1 Results Showing User-Rated Driving Abilities (on a scale of 1-5, with 5 being extremely easy).

Figure 9. Phase 1 Results Summary 


\subsection{User Preference}

The role of user preference is important to any usability assessment, and here perhaps even more so. Given the lack of experience with tilt controls in the general population, and a task that few have previously attempted (robot teleoperation), our hypothesis was conservative in assuming that users might prefer the virtual joystick regardless of performance outcomes. Joystick control was posited to be more familiar to users and a direct virtual mapping of current tactile controls. Lack of familiarity can sometimes doom even the most usable new systems, as consumer resistance to change is well-documented (Khan \& Hyunwoo, 2009; Ram \& Sheth, 1989). That said, ARL's study made clear that users had a number of issues with virtual joystick control; problems which the tilt controller was designed to overcome. Looking at numbers alone, it would appear to have done so, given the higher percentage of participants preferring tilt controls, but how is that preference tied to performance?

Table 2 shows that trial time was significantly faster for the joystick controls, and as one of three quantifiable measures of performance, it could be expected that users would summarize their own performance with Controller A superior to that of Controller B. Instead, survey results indicate the opposite-users rated their abilities superior with the tilt controls in all categories: move, obstacles, slow, med, hi, and drive. Additionally, while 18 users performed better with the joystick controller in terms of trial time, only nine preferred it. Examining the percent improvement between each user's best and worst controller trial averaged an $18 \%$ difference, regardless of whether a participant drove faster with Controller A or Controller B. This implies that preference is not obscured by percent improvement in trial times, as was assumed.

Instead, it seems to suggest that one or more of the other performance metrics ranked higher in users' own determination of their abilities. Participants were not informed of their trial times, nor were any stopwatches or clocks visible to them during task execution. However, knowledge of performance regarding path points hit and driving errors was more difficult to shield from users. While final numbers were not shared, users generally knew how many times they had collided with obstacles and could easily compare one run against another. Likewise, while participants could not always be certain that their robot ran over a path point, users almost certainly knew if they missed one entirely. Therefore, it makes sense that individuals might use those metrics to judge overall

performance as much, if not more so, than trial time. Of the nine users who drove faster with the joystick yet preferred the tilt controls, eight of them committed less minor errors with Controller B. Path points indicated less relationship to preference, as only three of nine users improved their driving accuracy with tilt-based controls; five hit fewer path points, and one user's performance held constant. Finally, both the NASA TLX and SUS results did indicate a relationship to preference when performance metrics alone could not explain a user's choice. It appears as if most users preferred the controller that yielded the lower workload score and higher usability ratings (both, again, hidden from the user). Ten users reported higher workload and lower SUS scores with Controller $\mathrm{B}$, and of those, only three still stated they preferred the tilt controls. Clearly, no one variable is enough to predict a user's preference, but this provides some insight as to how users rate the various performance metrics in their own assessments.

\section{Results \& Discussion - Phase 2}

The independent variable in Phase 2 was the controller type, C (tilt to drive; joystick camera) or D (tilt to drive; tilt-based camera), and once again a one-factor experiment with two levels. The dependent variables used for statistical analysis were updated to include task-specific qualitative feedback but still relied upon the standard performance measures-time, errors, and path points struck. Analysis of variance was repeated to examine the effect of the independent variable on the identified 14 
dependent variables. In this phase, none of these relationships proved significant (see Table 3), leading to a failure to reject the null hypothesis. This implies that tilt-based controls are not significantly better (or worse than) joystick-based controls when operating multiple robot degrees of freedom.

Table 3: The F-Statistic: Phase 2

\begin{tabular}{|r|r|r|r|r|r|r|r|r|}
\hline \multicolumn{2}{|c|}{} & \multicolumn{1}{c|}{ ptime } & \multicolumn{1}{c|}{ ttime } & majerror & minerror & pathpts & localization & \multicolumn{1}{c|}{ tlx } \\
\hline \multirow{3}{*}{ Controller C } & mean $(\bar{x})$ & 193.00 & 539.96 & 0.04 & 3.32 & 25.44 & 7.2 & 44.72 \\
\cline { 2 - 9 } & std dev $\left(s_{d}\right)$ & 140.31 & 154.64 & 0.2 & 1.8 & 3.42 & 1.58 & 13.93 \\
\hline \multirow{2}{*}{ Controller D } & mean $(\bar{x})$ & 152.32 & 556.56 & 0.12 & 3.12 & 26.08 & 6.64 & 46.07 \\
\cline { 2 - 10 } & std dev $\left(s_{d}\right)$ & 94.08 & 124.75 & 0.33 & 1.99 & 3.7 & 1.96 & 14.73 \\
\hline & $p(<0.05)$ & 0.228 & 0.584 & 0.327 & 0.639 & 0.227 & 0.223 & 0.393 \\
\hline$F$ & 1.53 & 0.31 & 1 & 0.23 & 1.54 & 1.57 & 0.76 \\
\hline \hline \multirow{2}{*}{ Controller C } & mean $(\bar{x})$ & 77.40 & 4.00 & 4.08 & 3.72 & 4.04 & 4.48 & 4.13 \\
\cline { 2 - 10 } & std dev $\left(s_{d}\right)$ & 12.47 & 1.08 & 0.99 & 0.79 & 0.84 & 0.77 & 0.54 \\
\hline \multirow{2}{*}{ Controller D } & mean $(\bar{x})$ & 75.10 & 4.12 & 4.21 & 4.00 & 3.96 & 4.44 & 4.04 \\
\cline { 2 - 10 } & std dev $\left(s_{d}\right)$ & 13.12 & 0.78 & 0.83 & 0.82 & 0.93 & 0.82 & 0.86 \\
\hline & $p(<0.05)$ & 0.389 & 0.600 & 0.524 & 0.183 & 0.714 & 0.814 & 0.479 \\
\hline & $F$ & 0.77 & 0.28 & 0.42 & 1.88 & 0.14 & 0.06 & 0.52 \\
\hline
\end{tabular}

\subsection{Performance}

Practice time notably decreased compared to Phase 1, mostly attributed to learning effects. Users were already acquainted with the tilt-based driving controls, and aside from a brief reintroduction to the course, they spent most of their hands-on practice familiarizing themselves with the task conditions and camera controls. Trial times were very similar for the two controllers, failing to achieve significance $\left(\bar{x}_{C}=540 s, \bar{x}_{D}=557 \mathrm{~s}\right)$. Likewise, the pathpts variable failed to reject the null hypothesis $\left(\bar{x}_{C}=25.44, \bar{x}_{D}=26.08\right)$; it did, however, decrease substantially from Phase 1 , where 28 points were found, on average, across 50 trials. Users struggled with the increased task complexity of Phase 2, and the shared priorities of speed, driving accuracy, avoidance of collisions, and visual identification tasks led to degraded performance in the path points variable. Surprisingly, this was the only performance area that suffered between the two phases, as both error variables (majerror and minerror) exhibited improved driving precision.

Within Phase 2, performance conditions were not expected to vary greatly, as the driving task remained the more time intensive portion of the trial and stayed constant between Controller $\mathrm{C}$ and D. Many users were able to identify the objects from their robot's position in each scanning box in approximately one minute, devoting about three minutes per trial to visual identification tasks. In cases where users struggled to find an object, they were asked to continue along the course after three minutes had elapsed in one location. For some individuals, a non-trivial amount of time was then spent identifying the location of the found object for mapping; this differed greatly from person to person

localization describes the dependent variable measuring the accuracy with which users identified where items within the robot's view were positioned in space. Multiple user comments implied that localization was equally difficult with both controllers, with a mixture of users indicating a clear 
preference for one over the other. In the case of camera pan/tilt, a visually intensive task, a visual representation of the camera's position (e.g. joystick location from center) might seemingly satisfy users more than the physical representation (e.g. degree of tilt) provided by the attitude-aware controls, but user-rated looking, which answered the question "identify which direction the camera is looking with respect to the robot," demonstrates otherwise $\left(\bar{x}_{C}=3.72, \bar{x}_{D}=4.00\right)$. Interestingly, the value of localization (performance based) slightly favored the joystick control and is not strongly associated with the looking variable. Similar to the phenomenon observed in Phase 1, users' own assessments of their abilities and preferences did not relate closely to performance results!

\subsection{User Preference}

Survey results indicate that 14 users preferred the joystick camera controls, while the remaining 11 preferred the tilt-based camera controls. Results from Phase 1 implied that trial time was not the best indicator of user preference, and results here support that conclusion. Thirteen users completed their trial faster with Controller C, and 12 with Controller D, with an average trial time improvement of $21 \%$. However, 13 users also preferred the controller on which they did not perform most quickly. Six of those participants performed best with Controller D, yet preferred Controller C, while the other seven participants did the opposite. Neither pathpts nor the error variables showed a relationship to user preference; likewise, TLX and System Usability Scores, partly due to a lower variance in those values in Phase 2 trials. Instead, preference appears more closely related to the order in which controllers were presented to the user. While theoretically this ordering effect was accounted for by counterbalancing presentation of the independent variables, in the case of user preference, it cannot be wholly eliminated. Twenty users preferred the controller they used last, indicating that learning effects, perhaps magnified due to the control similarities, did play a role in user preference.

The pan, tilt, and scanning variables' results slightly favored the tilt-based camera controls, with a number of users describing them as "smoother." Joystick controls were described as "intuitive" and "less physical," contributing to lower mental workload scores, although several individuals complained that the camera movement should be slowed down considerably. None of these preferences were dramatic enough to prove statistically significant.

\subsection{Mode Confusion}

Aside from determining user performance and satisfaction with each of the two controllers, Phase 2 was additionally designed to test the suitability of tilt-based controls for two dissimilar robot actions (driving and camera manipulation). In complex control interfaces, it is not uncommon for users to encounter confusion regarding which controls are currently active and which buttons and gestures result in the outcome they are expecting-a form of mode confusion. This research raises a question asking whether using the same control mode to operate distinctly different robot actions eases this confusion or exacerbates it. Controller $\mathrm{C}$ presented a situation where two control modes (joystick and tilt) separated and defined the two robot actions they controlled (camera and driving), while Controller D used a single control mode, accessed through two separate activation buttons (deadman switches) to control both robot camera and driving via tilt inputs.

To capture any effects due to mode confusion, users were each asked, in post-iteration surveys, whether they encountered any mode confusion in their trials. Users could respond never, rarely, sometimes, very often, or always. These instances of confusion could include activating the wrong part of the robot (e.g., moving the camera when the user intended to drive) or confusion regarding input mode (e.g., trying to drag the deadman switch like a joystick). Those that answered anything but never were then asked to rate how often the confusion occurred, as a percent of all control activations. Fig. 10 graphically illustrates these results. 
Walker et al., User-Centered Design of an Attitude-Aware Controller for Ground Reconnaissance Robots

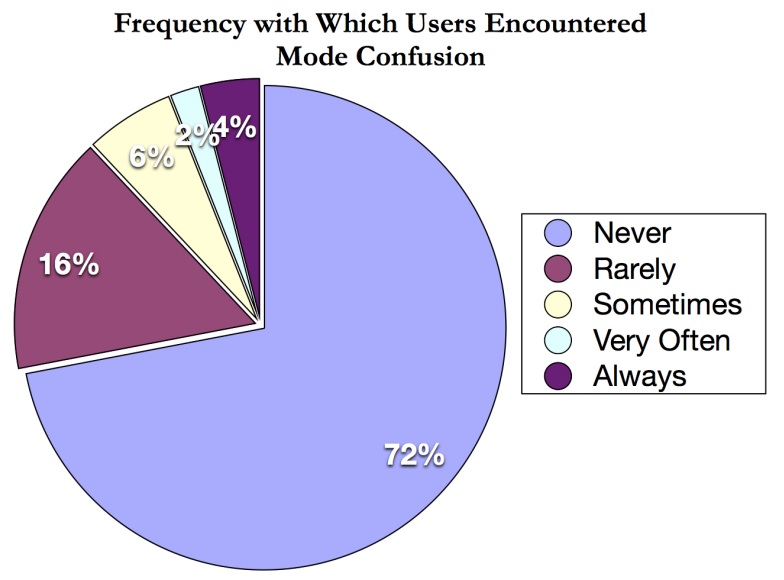

(a) Frequency With Which Mode Confusion Occurred

$\%$ of the Time this Mode Confusion Occurred

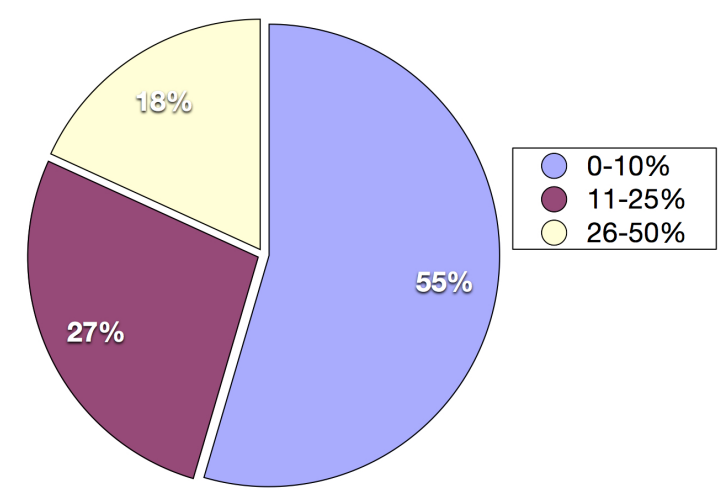

(b) Mode Confusion as a Percent of All Control Activations

Figure 10. Mode Confusion Charts 
Eight individuals reported some mode confusion with Controller $\mathrm{C}$, while six individuals reported confusion with Controller D. Four of these individuals reported confusion with both controller types. Severity of confusion does not appear to be significant, and frequency of confusion remained low. In all, mode confusion appears much more closely related to the user than it does the controller. User-reported technical proficiency indicated no deficiency on the part of the four participants who experienced persistent mode confusion; however, their gaming experience ranks less adept than the participant population, on average. Users rated their skills with video game systems (e.g., the XBox) and smartphone games on a scale from no experience (1) to excellent (5). The sample population averaged 3.38 on video game skills (slightly better than average), while the four participants examined here averaged 3, right at average. On smartphone game skills, the disconnect was even greater, with the sample population reporting a 3.28 average and the participant subset averaging just 2.5 (between poor and average). Gaming skills have been previously linked to teleoperation abilities (J. Y. C. Chen, 2010); however, their relation to mode confusion, specifically, has not been documented.

\section{Conclusion}

The purpose of the research presented here was to assess the usability of attitude-aware controls as an alternative to more commonly employed, tactile operator control unit methodologies. The goal was to create a controller prototype that promoted simple, efficient control of small reconnaissance robots used by dismounted ground forces during contingency operations while overcoming the challenges of touchscreen use. Smartphones offer a powerful, lightweight, multimodal platform for control and have the potential to become highly adaptable, lightweight battlefield tools, assuming usability issues specific to field operations can be addressed.

The sum of our experiment results indicate that tilt-based controls are suitable for teleoperating small ground robots and provide superior user experiences (in terms of satisfaction) in many instances. While not all performance metrics favored the tilt controls, over the course of three experiment phases (or roughly 4.5 hours of robot familiarization), all users were able to function effectively with an unfamiliar control mode leading to a lack of statistical significance when compared to virtual joystick controls. While not a rousing endorsement, the fact that tilt controls are no worse than other touchscreen-based implementations moves us one step closer to improving the amount of control available to over-tasked users operating in austere environments. Its great benefit is that it can be employed on a device that is already being carried by soldier-operators, improving interoperability and decreasing the size and weight of the solider load-one of the crucial (and often overlooked) aspects of any technology designed for the warfighter. By implementing a proprioceptive control mode on a device that is customarily considered virtual, tilt-based control provides a reliable method of operating smartphones while gloved. The less visual nature of the control also permits a better distribution of operator attention between controlling the robot and remaining situationally aware. This effect has not yet been proven but may be evaluated in later experiments with Army users. For now, experiment results support that both camera and driving tasks could be managed satisfactorily with attitude-aware controls, promoting their continued development for said purpose.

Summarizing, this usability experiment proved that better field-deployable smartphone control implementations do exist, rendering virtual joystick alternatives less desirable. Attitude-aware controls overcome many of the user complaints from Pettitt's 2011 experiment at ARL, including lack of haptic feedback (Pettitt et al., 2011). The question now remains how attitude-aware controls would compare to the tactile (XBox 360) controls currently in use with the Army's PackBot systems. This comparison would implement more realistic tasks and conditions, specifically, by testing with military users to better understand for whom we are designing while providing a unique perspective on 
Walker et al., User-Centered Design of an Attitude-Aware Controller for Ground Reconnaissance Robots

the balance of function and form, appreciating that everything used is carried.

\section{References}

Antal, J. (2009). I fight the body electric! Military Technology, 33(7), 22 - 30.

Apple. (2011, October). Core motion framework reference. Retrieved from http:// developer.apple.com/library/ios/\#documentation/CoreMotion/Reference/ CoreMotion_Reference/_index.html.

Apple. (2014, December). iPhone 6 technical specifications. Retrieved from www . apple.com/i phone-6/ specs.

Applied Research Associates. (2011). Pointman: Purpose-built for tactical missions. Retrieved from http: // www.ara.com/robotics/Small-Unmanned-Ground-Vehicle.html.

Army Science Board. (2002). Ad hoc study on human robot interface issues. Technical Report.

Bland, E. (2008, December). Wii-controlled robots made for combat. Discovery News, MSNBC.com.

Blitch, J. G. (2003). Adaptive mobility for rescue robots. In E. M. Carapezza (Ed.), Proceedings of SPIE, Sensors and Command, Control, Communications and Intelligence (C3I) Technologies for Homeland Defense and Law Enforcement II (Vol. 5071). doi: 10.1117/12.503975.

Brooke, J. (1996). SUS: A quick and dirty usability scale. In P. Jordan, B. Thomas, B. Weerdmeester, \& I. McClelland (Eds.), . Taylor and Francis.

Carlson, J., \& Murphy, R. R. (2005). How UGVs physically fail in the field. In IEEE Transactions on Robotics (Vol. 21, p. 423 - 437). doi: 10.1109/TRO.2004.838027.

Center for Army Lessons Learned, Task Force Devil. (2003, May). The modern warrior's combat load: Dismounted operations in Afghanistan (April-May 2003).

Chen, J. Y. C. (2010). UAV-guided navigation for ground robot tele-operation in a military reconnaissance environment. Ergonomics, 53(8), pp. 940-950. doi: 10.1080/00140139.2010.500404.

Chen, J. Y. C., Haas, E. C., \& Barnes, M. J. (2007, November). Human performance issues and user interface design for teleoperated robots. In IEEE Transactions on Systems, Man, and Cybernetics-Part C: Applications and Reviews (Vol. 37). doi: 10.1109/TSMCC.2007.905819.

Chen, T., Yesilada, Y., \& Harper, S. (2010). What input errors do you experience? Typing and pointing errors of mobile web users. International Journal of Human Computer Studies, 68, 138-157. doi: 10.1016/j.ijhcs.2009.10.003.

Chong, V. V.-K. (2000). Heuristics for mitigating mode confusion in digital cameras (Unpublished master's thesis). University of Victoria, Victoria, British Columbia.

Cockburn, A. (1997a, September-October). Structuring use cases with goals: Part 1. Journal of Object Oriented Programming, 35-40.

Cockburn, A. (1997b, November-December). Structuring use cases with goals: Part 2. Journal of Object Oriented Programming, 56-62.

Conway, J., \& Hillegass, A. (2010). iPhone programming: The big nerd ranch guide. Indianapolis, IN: Pearson Technology Group.

Dension. (2011). WiRC user's manual v2.0 (v2.0 ed.). Retrieved from http://www.wirc.dension . com/support.

Department of the Army. (1990, June). Field manual 21-18: Footmarches. U.S. Department of the Army, USA.

Department of the Army. (2013, June). TRADOC regulation 71-20. U.S. Department of the Army, USA.

Donmez, B., Pina, P. E., \& Cummings, M. L. (2008). Evaluation criteria for human-automation performance metrics. In Proceedings of the Performance Metrics for Intelligent Systems Workshop. doi: 10.1145/1774674.1774687.

Erwin, S. I. (2001, October). Army's future tactical net apt for high-speed combat. National Defense. Retrieved from http://www.nationaldefensemagazine.org/archive/2001/October/ Pages/Armys_Future6935.aspx.

Fong, T., \& Thorpe, C. (2001). Vehicle teleoperation interfaces. Autonomous Robots, 11, 9-18.

Fong, T., Thorpe, C., \& Baur, C. (2003). Multi-robot remote driving with collaborative control. In IEEE Transactions on Industrial Electronics (Vol. 50, p. 699 - 704). doi: 10.1109/TIE.2003.814768. 
Walker et al., User-Centered Design of an Attitude-Aware Controller for Ground Reconnaissance Robots

Fung, N. (2011). Light weight, portable operator control unit using an Android-enabled mobile phone. In D. W. Gage \& C. M. Shoemaker (Eds.), Unmanned systems technology XIII, Proceedings of SPIE (Vol. 8045). doi: 10.1117/12.883308.

Hart, S., \& Staveland, L. (1988). Development of NASA-TLX (Task load index: Results of empirical and theoretical research. In P. Hancock \& N. Meshkati (Eds.), Human mental workload (p. 139-183). Elsevier. doi: 10.1016/S0166-4115(08)62386-9.

Hillegass, A. (2011). Objective-c programming: The big nerd ranch guide. Indianapolis, IN: Pearson Technology Group.

Hinckley, K., Pierce, J., Sinclair, M., \& Horvitz, E. (2000). Sensing Techniques for Mobile Interaction. In Symposium on User Interface Software and Technology, CHI Letters (Vol. 2, p. 91-100). doi: 10.1145/354401.354417.

Human Performance Research Group, NASA. (1988). NASA task load index (TLX) v 1.0: Pen and pencil package. Retrieved from http://humansystems.arc.nasa.gov/groups/TLX/paperpencil .html.

International Organization for Standardization (ISO). (2008). Ergonomics of human-system interaction - multiple parts (Norm No. ISO 9241). Retrieved from http://www.iso.org/iso/home/store/ catalogue_tc/catalogue_tc_browse.htm?commid=533N72. Geneva, Switzerland: ISO.

iRobot Corporation. (2012). iRobot 110 FirstLook. Retrieved from http://www.irobot.com/us / learn/defense/firstlook.aspx.

Israelski, E., \& Lund, A. M. (2003). The human-computer interaction handbook. In J. A. Jacko \& A. Sears (Eds.), (pp. 772-789). Hillsdale, NJ: L. Erlbaum Associates Inc. Retrived from http://dl . acm .org/citation.cfm?id=772072.772121.

Jang, I., \& Park, W. (2004). A gesture-based control for handheld devices using accelerometer. In Proceedings of the 9th Iberoamerican Congress on Pattern Recognition (Vol. 3287, p. 259-266).

Jokela, T., Iivari, N., Matero, J., \& Karukka, M. (2003). The standard of user-centered design and the standard definition of usability: Analyzing ISO 13407 against iso 9241-11. In Proceedings of the Latin American Conference on Human-Computer Interaction (p. 53-60). New York, NY: ACM. doi: $10.1145 / 944520.944525$.

Keyes, B., \& Yanco, H. A. (2006). Camera placement and multi-camera fusion for remote robot operation. In IEEE International Workshop on Safety, Security and Rescue Robotics.

Khan, K., \& Hyunwoo, K. (2009). Factors affecting consumer resistance to innovation: A study of smartphones (Unpublished master's thesis). Jonkoping International Business School, Jonkoping, Sweden.

Knox, M. (2012, December). Interview with CPT Michael Knox, U.S. Army. E-mail Correspondence.

Lane, J. C., Carignan, C. R., Sullivan, B. R., Akin, D. L., Hunt, T., \& Cohen, R. (2002). Effects of time delay on telerobotic control of neutral buoyancy vehicles. In Proceedings of the 2002 IEEE International Conference on Robotics and Automation.

Lankenau, A. (2001). Avoiding mode confusion in service robots - The bremen autonomous wheelchair as an example. In Proceedings of the 7th International Conference on Rehabilitation Robotics (ICORR 2001) (p. 162-167). Evry, France.

Ling, C., Hwong, W., \& Savendy, G. (2007, March-April). A survey of what customers want. Behaviour and Information Technology, 26(2), 149-163, doi: 10.1080/01449290500128214.

Loper, M., Koenig, N., Chernova, S., Jones, C., \& Jenkins, O. (2009). Mobile human-robot teaming with environmental tolerance. In Proceedings of the 4th ACM/IEEE International Conference on HumanRobot Interaction (p. 157-164), doi: 10.1145/1514095.1514124.

McGovern, D. E. (1987). Experiences in teleoperation of land vehicles. Technical Report. Sandia National Labs: Albuquerque, NM.

McHale, J. (2014). Smartphones on the battlefield. Retrieved from http://mil-embedded.com/ articles/smartphones-the-battlefield/.

Milian, M. (2011, July). U.S. Army may soon equip troops with smartphones. Retrieved from http://www.cnn.com/2011/TECH/mobile/07/12/army.smartphones/ index.html?hpt=hp_t 2 .

Morrow, J. (2012, December). Interview with CPT Jack Morrow, U.S. Army. E-mail Correspondence. 
Walker et al., User-Centered Design of an Attitude-Aware Controller for Ground Reconnaissance Robots

Murphy, R. R. (2000). Introduction to AI Robotics. Cambridge, MA: The MIT Press.

Oakley, I., \& O'Modhrain, S. (n.d.). Tilt to scroll: Evaluating a motion based vibrotactile mobile interface. In Proceedings of the Eurohaptics Conference, 2005 and Symposium on Haptic Interfaces for Virtual Environment and Teleoperator Systems, 2005. World Haptics 2005. First Joint (p. 40-49). doi: 10.1109/WHC.2005.138.

Oakley, I., \& Park, J. (2007). A motion-based marking menu system. In Proceedings of CHI '07 Extended Abstracts on Human Factors in Computing Systems (pp. 2597-2602). New York, NY: ACM. doi: 10.1145/1240866.1241048. doi:blah10.1145/1240866.1241048

Okafor, A. (2010, July). Parade of Rain Blog. Retrieved from http://www.paradeofrain.com/ 2010/07/lessons-learned-in-tilt-controls/.

Orbotix. (2012). iOS developer quick start guide. ReadMe in open source SDK. Retrieved from https : // github.com/orbotix/Sphero-iOS-SDK.

Pettitt, R. A., Redden, E. S., Fung, N., Carstens, C. B., \& Baran, D. (2011, September). Scalability of robotic controllers: An evaluation of controller options-Experiment ii. Technical Report No. 5776.

Piskorski, S., Brulez, N., \& Eline, P. (2011, May). AR.Drone developer guide SDK 1.7. Retrieved from https: / / projects.ardrone.org.

Pitman, D. (2010). Collaborative micro aerial vehicle exploration of outdoor environments (Unpublished master's thesis). Massachusetts Institute of Technology, Cambridge, MA.

Pitman, D., \& Cummings, M. L. (n.d.). Collaborative exploration with a micro aerial vehicle: A novel interaction method for controlling a MAV with a hand-held device. Advances in Human-Computer Interaction, 2012, doi: 10.1155/2012/768180.

Qua, A. (n.d.). Cube runner. Retrieved from http://andyqua.co.uk/CubeRunner/Welcome .html.

Rahman, M., Gustafson, S., Irani, P., \& Subramanian, S. (2009). Tilt techniques: Investigating the desterity of wrist-based input. In Proceedings of the IEEE International Conference on Computer Human Interaction. doi: 10.1145/1518701.1518997.

Ram, S., \& Sheth, J. N. (1989). Consumer resistance to innovations: The marketing problem and its solutions. Journal of Consumer Marketing, 6(2), 5-14. doi: 10.1108/EUM0000000002542.

Recon Robotics. (2012, May). Throwbot XT - With audio. Retrieved from http: / / www.reconrobotics .com/products/Throwbot_XT_audio.cfm.

Redden, E. S., Elliott, L. R., Pettitt, R. A., \& Carstens, C. B. (2011, June). Scaling robotic systems for dismounted warfighters. Journal of Cognitive Engineering and Decision Making, 5(2), 156-185. doi: $10.1177 / 1555343411409315$.

Rekimoto, J. (1996). Tilting operations for small screen interfaces. In Proceedings of the 9th Annual ACM Symposium on User Interface Software and Technology. doi: 10.1145/237091.237115.

Scalesse, C. (2013, January). Tutorial: Accelerometer calibration and optimizations. Retrieved from http://iphonedevsdk.com/forum/iphone-sdk-tutorials/39833-tutorial -accelerometer-calibration-optimizations.html.

Shneiderman, B., \& Plaisant, C. (2010). Designing the user interface. Boston, MA: Addison-Wesley.

Tilbury, D., \& Ulsoy, G. A. (2011). A new breed of robots that drive themselves. Mechanical Engineering, 133(2), 28.

Tomlinson, Z. A. (2009). Influence of spatial ability on primary and secondary space telerobotics operator performance. Aviation, Space, and Environmental Medicine, 80, 221.

Tracey, M. R., \& Lathan, C. E. (2001). The interaction of spatial ability and motor learning in the transfer of training from a simulator to a real task. Studies in Health Technology and Informatics, 81, 521 - 527.

University of Kent Careers and Employability Service. (2013). Non-Verbal Reasoning Test. Retrieved from http://www.kent.ac.uk/careers/tests/spatialtest.htm.

van Erp, J. B. (1999, April). Controlling unmanned vehicles: The human factors solution. In RTO SCI Symposium on "Warfare Automation: Procedures and Techniques for Unmanned Vehicles". Ankara, Turkey.

van Erp, J. B., \& Padmos, P. (2003). Image parameters for driving with indirect viewing systems. Ergonomics, 46(15), 1471-1499. doi: 10.1080/0014013032000121624. 
Walker et al., User-Centered Design of an Attitude-Aware Controller for Ground Reconnaissance Robots

Walker, A. M. (2013). Attitude-aware smartphones for teleoperated robot control (Unpublished doctoral dissertation). University of Oklahoma, Norman, OK.

Wang, J., Lewis, M., \& Hughes, S. (2004). Gravity-referenced attitude display for teleoperation of mobile robots. In Proceedings of the Human Factors and Ergonomics Society 48th Annual Meeting. doi: $10.1177 / 154193120404802312$.

Winfield, A. F. (2000). Future directions in tele-operated robotics. Technical Report. Bristol, UK: University of the West of England.

Yagoda, R. E., \& Hill, S. G. (2011). Using mobile devices for robotic controllers: Examples and some initial concepts for experimentation (No. ARL-TN-436). Technical Report No. ARL-TN-436. Army Research Laboratory.

Yarrish, G. (2011, January). Look, ma! No radio! Retrieved from ModelAirplaneNews.com.

MAJ Amber M. Walker, United States Military Academy, Department of Civil and Mechanical Engineering, West Point, NY USA. Email: amber.walker@usma.edu. David P. Miller, University of Oklahoma, School of Aerospace and Mechanical Engineering, Norman, OK USA. Email: dpmiller@ou . edu. Chen Ling, University of Akron, Systems Engineering and Mechanical Engineering Department, Akron, OH USA. Email: cl99@akron. edu. 